Review

\title{
Real-time fMRI neurofeedback: Progress and challenges
}

\author{
J. Sulzer $^{\text {a,*}}$, S. Haller ${ }^{\text {b,**, F. Scharnowski }}{ }^{\text {c,d,***, N. Weiskopf }}{ }^{\text {e,*****, N. Birbaumer }}{ }^{\text {f,g }}$, M.L. Blefari ${ }^{\text {a }}$,
} A.B. Bruehl ${ }^{\text {h }}$, L.G. Cohen ${ }^{\text {i }}$, R.C. deCharms ${ }^{j}$, R. Gassert ${ }^{a}$, R. Goebel ${ }^{k}$, U. Herwig ${ }^{\text {h,l }}$, S. LaConte ${ }^{m}$, D. Linden ${ }^{n}$, A. Luft $^{\mathrm{o}, \mathrm{p}}$, E. Seifritz ${ }^{\mathrm{h}}$, R. Sitaram ${ }^{\mathrm{f}, \mathrm{q}, \mathrm{r}, * * * * *}$

a Department of Health Sciences and Technology, Swiss Federal Institute of Technology, (ETH), Zurich CH-8092, Switzerland

${ }^{\mathrm{b}}$ University of Geneva, Geneva University Hospital CH-1211, Switzerland

${ }^{c}$ Department of Radiology and Medical Informatics - CIBM, University of Geneva, Switzerland

d Institute of Bioengineering, Swiss Institute of Technology Lausanne (EPFL) CH-1015, Switzerland

e Wellcome Trust Centre for Neuroimaging, UCL Institute of Neurology, University College London WC1E 6BT, UK

${ }^{\mathrm{f}}$ The Institute of Medical Psychology and Behavioral Neurobiology, University of Tuebingen 72074, Germany

g Ospedale San Camillo, IRCCS, Venice 30126, Italy

${ }^{h}$ Department of Psychiatry, Psychotherapy and Psychosomatica, Zürich University Hospital for Psychiatry, Zurich CH-8032, Switzerland

${ }^{i}$ National Institutes of Health, Bethesda 20892, USA

j Omneuron, Inc., Menlo Park 94025, USA

${ }^{k}$ Department of Neurocognition, University of Maastricht 6200, The Netherlands

${ }^{1}$ Department of Psychiatry and Psychotherapy III, University of Ulm, Germany

${ }^{\mathrm{m}}$ Virginia Tech Carilion Research Institute 24016, USA

${ }^{\mathrm{n}}$ Cardiff University CF10 3AT, UK

${ }^{\circ}$ Department of Neurology, University Hospital Zurich, Switzerland

p University of Zurich $\mathrm{CH}-8008$, Switzerland

${ }^{\mathrm{q}}$ Department of Biomedical Engineering, University of Florida, Gainesville 32611, USA

${ }^{\mathrm{r}}$ Sri Chitra Tirunal Institute of Medical Science and Technology, Trivandrum, India

\section{A R T I C L E I N F O}

\section{Article history:}

Accepted 19 March 2013

Available online 27 March 2013

\section{Keywords:}

Real-time fMRI

Neurofeedback

Operant conditioning

Brain-computer interface

\begin{abstract}
A B S T R A C T
In February of 2012, the first international conference on real time functional magnetic resonance imaging (rtfMRI) neurofeedback was held at the Swiss Federal Institute of Technology Zurich (ETHZ), Switzerland. This review summarizes progress in the field, introduces current debates, elucidates open questions, and offers viewpoints derived from the conference. The review offers perspectives on study design, scientific and clinical applications, rtfMRI learning mechanisms and future outlook.
\end{abstract}

(c) 2013 Elsevier Inc. All rights reserved.

\section{Contents}

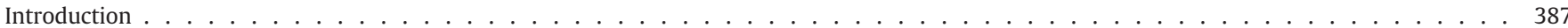

Considerations in study design $\ldots \ldots \ldots \ldots$

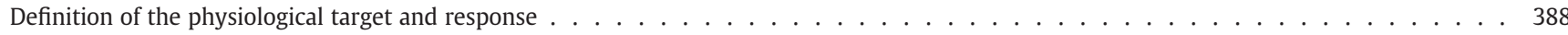

Neurofeedback of physiological target response . . . . . . . . . . . . . . . . . . . . . . . . . . . . . . 389

Neurofeedback signal. . . . . . . . . . . . . . . . . . . . . . . . . . . . . . . 389

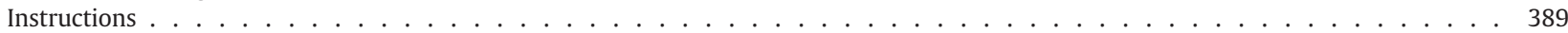

Task design . . . . . . . . . . . . . . . . . . . . . . . . . . . . . . . . . . . 389

\footnotetext{
* Correspondence to: J. Sulzer, Leonhardstrasse 27 B9.2, Zurich CH-8092, Switzerland.

** Correspondence to: S. Haller, Rue Gabrielle Perret-Gentil 4, Geneva CH-1211, Switzerland.

*** Correspondence to: F. Scharnowski, EPFL, IBI-STI, GRVDV, BM4.129, Station 17, Lausanne CH-1015, Switzerland.

**** Correspondence to: N. Weiskopf, 12 Queen Square, London WC1N 3BG, UK.

***** Correspondence to: R. Sitaram, Department of Biomedical Engineering, University of Florida, Gainesville 32611, USA.

E-mail addresses: jamessulzer@gmail.com (J. Sulzer), sven.haller@hcuge.ch (S. Haller), scharnowski.frank@gmail.com (F. Scharnowski), n.weiskopf@ucl.ac.uk (N. Weiskopf), ranganatha.sitaram@bme.ufl.edu (R. Sitaram).
} 
Transfer after successful training . . . . . . . . . . . . . . . . . . . . . . . . . . . . . . . . . . . . . 390

Experimental control conditions. . . . . . . . . . . . . . . . . . . . . . . . . . . . . . . . . . . . . 390

Behavioral effects of neurofeedback . . . . . . . . . . . . . . . . . . . . . . . . . . . . . . . . . . . . . . . . . . 390

Scientific applications . . . . . . . . . . . . . . . . . . . . . . . . . . . . . . . . . . . . . . . . . . . . . 391

Clinical applications . . . . . . . . . . . . . . . . . . . . . . . . . . . . . . . . . . . . . . . . 392

Which neural circuit to train?. . . . . . . . . . . . . . . . . . . . . . . . . . . . . . . . . . . 392

Potential risks . . . . . . . . . . . . . . . . . . . . . . . . . . . . . . . . . . . . . . . . . . 393

Determining effect size . . . . . . . . . . . . . . . . . . . . . . . . . . . . . . . . . . 393

Randomized controlled trials and multicenter studies. . . . . . . . . . . . . . . . . . . . . . . . . . . . . . . . . 393

Replication .. . . . . . . . . . . . . . . . . . . . . . . . . . . . . . . . . . . 393

Learning mechanisms . . . . . . . . . . . . . . . . . . . . . . . . . . . . . . . . . . . . . . . . . . . 394

What are the learning mechanisms engaged by neurofeedback?. . . . . . . . . . . . . . . . . . . . . . . . . . . . . . . 394

What are the psychobiological mechanisms of neurofeedback? . . . . . . . . . . . . . . . . . . . . . . . . . . . . . . . . 394

Are there limits to operant learning and how can they be overcome? . . . . . . . . . . . . . . . . . . . . . . . . . . . . . . 395

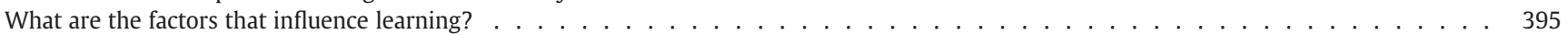

Where is the future of rtfMRI neurofeedback? . . . . . . . . . . . . . . . . . . . . . . . . . . . . . . . . . . . . . . . . . 395

Immediate future . . . . . . . . . . . . . . . . . . . . . . . . . . . . . . . . . . . . . . 395

The longer term future . . . . . . . . . . . . . . . . . . . . . . . . . . . . . . . . . . . . . 396

Conclusions from the meeting . . . . . . . . . . . . . . . . . . . . . . . . . . . . . . . . . . . . . . . 396

Acknowledgments . . . . . . . . . . . . . . . . . . . . . . . . . . . . . . . . . . . . . . . . . . . . 397

Appendix A. List of presenters (alphabetical order) . . . . . . . . . . . . . . . . . . . . . . . . . . . . . . . . . . . . . . 397

References. . . . . . . . . . . . . . . . . . . . . . . . . . . . . . . . . . . . . . . . 397

\section{Introduction}

On February 16th and 17th, 2012, approximately 150 international researchers joined the first conference on an emerging discipline known as real-time functional magnetic resonance imaging (rtfMRI) neurofeedback at the Swiss Federal Institute of Technology in Zurich (ETHZ), Switzerland (www.relab.ethz.ch/rtfMRI2012). The purpose of this meeting was to provide a forum to share progress and discuss the challenges for future research and clinical applications. The meeting also inspired the creation of the following work, which reviews current progress and introduces open questions and controversies.

Functional MRI measures the blood oxygenation level dependent (BOLD) signal in the brain (Ogawa et al., 1990a,1990b), a quantity that arises from several biophysical and physiological sources (Kim and Ogawa, 2012) and represents a vascular coupling to neural activity (Logothetis, 2008; Logothetis et al., 2001). FMRI provides specific advantages over other non-invasive neuroimaging methods such as electroencephalographic recordings (EEG), including whole brain coverage and finer spatial resolution on the order of several millimeters. We define rtfMRI, first published by Cox et al. (1995), as any process that uses functional information from a MRI scanner where the analysis and display of the fMRI keep pace with data acquisition. Although whole brain fMRI data sampling can now be performed in around half a second (Feinberg et al., 2010), typical protocols still use sampling rates covering the brain approximately every two seconds. Cox et al. described that real-time brain mapping could be used for quality assurance, faster protocol development and "interactive experimental paradigms". At present, rtfMRI has additionally been applied to intraoperative surgical guidance (Hirsch et al., 2000), brain-computer interfaces (BCIs) (Sorger et al., 2012; Yoo et al., 2004), and neurofeedback.

While EEG neurofeedback has a long history (Elbert et al., 1980; Lynch et al., 1974; Rockstroh et al., 1984, 1993), there has been a recent rise in attention to rtfMRI neurofeedback, providing a timely background for the conference. Fig. 1 shows that there were almost as many journal papers published on the topic in 2011-2012 ( $\mathrm{n}=73$ ) than the preceding ten years combined $(\mathrm{n}=75)$. The figure illustrates that recently neurofeedback and methods development currently comprise the plurality of the rtfMRI field, and as a result this paper focuses on neurofeedback approaches (Berman et al., 2011; Bray et al., 2007; Caria et al., 2007, 2010; Chiew et al., 2012; deCharms et al., 2004, 2005; Frank et al., 2012; Haller et al., 2010; Hamilton et al., 2011;
Hawkinson et al., 2011, 2012; Hinds et al., 2011; Johnson et al., 2012; Johnston et al., 2010, 2011; Lee et al., 2011, 2012; Li et al., 2012; Linden et al., 2012; McCaig et al., 2011; Posse et al., 2003; Rota et al., 2009; Scharnowski et al., 2012; Shibata et al., 2011; Subramanian et al., 2011; Sulzer et al.; Veit et al., 2012; Weiskopf et al., 2003, 2004a; Yoo and Jolesz, 2002; Yoo et al., 2008; Zotev et al., 2011). Fig. 1 also clearly shows that review papers regarding this technology are rather plentiful (e.g. Caria et al., 2012; Chapin et al., 2012; deCharms, 2007, 2008; LaConte, 2011; Linden, 2012b; Sitaram et al., 2010; Weiskopf, 2012; Weiskopf et al., 2004b). Therefore the purpose of this paper is to focus more on the open questions identified during the conference and the challenges that lie ahead. The paper is divided into five subsections that examine rtfMRI neurofeedback from different perspectives: 1) study design, 2) scientific applications, 3) clinical applications, 4) learning mechanisms and 5) the future of rtfMRI neurofeedback.

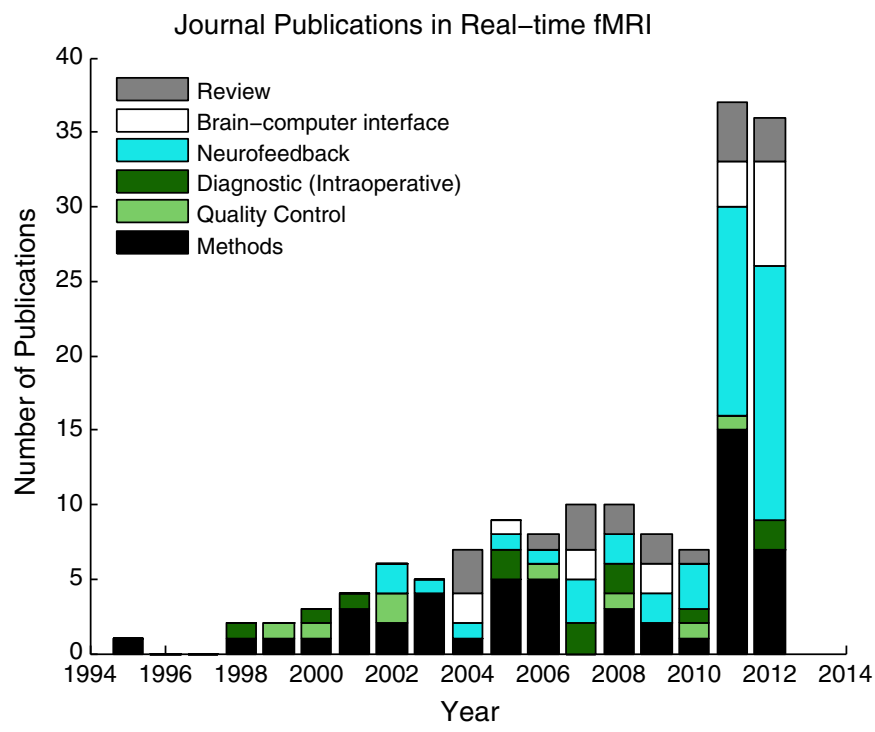

Fig. 1. Results of literature search regarding rtfMRI, found using the search term "Real-time fMRI" in Google Scholar, restricting findings to journal publications that use rtfMRI, develop technology specifically for rtfMRI or reviews primarily about rtfMRI. Publications were then categorized and color-coded accordingly. 


\section{Considerations in study design}

The design of a study depends on its objectives. The experimental objectives of neurofeedback studies may range from demonstrating neurofeedback induced learning of self-regulation to specific behavioral effects (e.g. Rota et al., 2009; Shibata et al., 2011) or clinical improvement in patients (e.g. deCharms et al., 2005; Ruiz et al., 2013; Subramanian et al., 2011). However, the majority of neurofeedback studies employ a similar experimental framework and schedule, primarily consisting of:

1. Definition of the physiological target and response: a region is anatomically specified or a functional localizer is applied to define the brain region, network and/or physiological response to be trained.

2. Neurofeedback of the physiological target response and measurement of subject performance: the participant is presented with online information on the activity of the physiological target to be trained (see Fig. 2) and attempts to learn to control the activation in the target brain area through the use of mental strategies, which can include anything from simple finger tapping to mental imagery or complex cognitive tasks. Feedback training may span several minutes, hours, or repeated sessions over days.

3. Transfer after successful training: when the participants have achieved successful regulation, they are tested to demonstrate whether they are able to maintain the skill of controlling brain activation or performing a task in the absence of feedback and/or in a different setting or task.

4. Experimental controls: studies employed different control groups or within subject control conditions to control for confounds in learning, behavioral and placebo effects.

5. Testing of behavioral effects: after participants learned effective regulation, one can test if this results in specific behavioral effects, typically before and after learning.

\section{Definition of the physiological target and response}

The definition of the neurofeedback target typically depends on the behavioral effect that is sought. For example, experiments that aimed at modulating reaction times manipulated the activity in motor areas such as the supplementary motor area (SMA) or primary motor cortex (M1) (Bray et al., 2007; Weiskopf et al., 2004a). Another experiment, aimed at changing pain perception, regulated activity in the rostral anterior cingulate cortex (rACC) (deCharms et al., 2005). Shibata et al. aimed at specific voxels in the primary and secondary visual cortices to evoke a change in visual perception (Shibata et al., 2011). Using previous knowledge of neural mechanisms underlying the desired behavioral change is the key to selecting the desired ROI.

The physiological target may be the average BOLD response in a chosen ROI, but it may also be more complex such as the differential activity in two ROIs (Chiew et al., 2012; Weiskopf et al., 2004b). Ongoing studies explore the possibility for feedback of connectivity between brain areas (e.g. presentations by Ruiz, and Zilverstand), similar to functional or effective connectivity measures used off-line (e.g. Biswal et al., 1995; Roebroeck et al., 2005). Specific ROIs can often be anatomically defined based on brain atlases or macroscopic anatomical landmarks, such as the insular cortex (Caria et al., 2007), but also functionally defined, such as the parahippocampal place area (Weiskopf et al., 2004a). A combination of overlaying functional activity on anatomical images may help further improve demarcation (e.g. hand knob of primary motor cortex presented by Blefari). Brain networks are usually difficult to define anatomically due to high variability. However, anatomical localizers may be more appropriate in certain cases where the relevant anatomical region is well defined and a reliable functional localizer is difficult (e.g. substantia nigra presented by Sulzer). Some unpublished evidence comparing functional to anatomical localizers for a given ROI was offered at the conference, showing that functional localizers offer a better contrast-tonoise signal in some circumstances, but that precise head stabilization
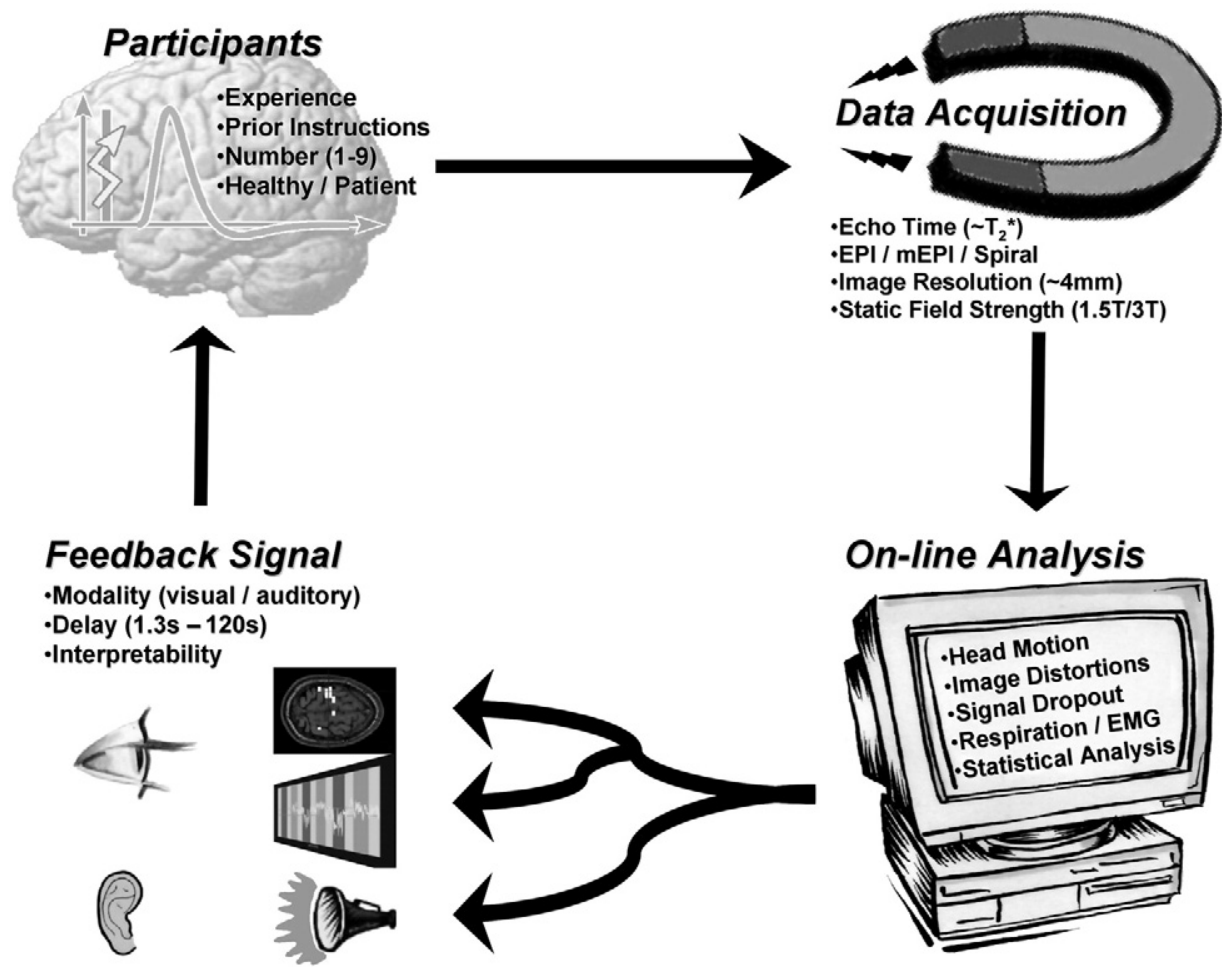

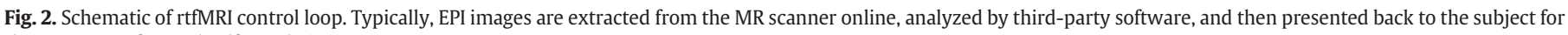
the purposes of neural self-regulation.

Reprinted from Weiskopf et al., 2004b with permission from Elsevier. 
is required to maintain this advantage as head movements of one millimeter or greater can remove this advantage over anatomical selection (presentation by deCharms). An alternative to general linear model (GLM)-based functional localizers is multivariate pattern analyses (MVPA) of BOLD responses, which allow the experimenter to identify complex and interacting activity patterns over the whole brain, or a prespecified set of regions, probably best reflecting network activity (LaConte, 2011; LaConte et al., 2007).

\section{Neurofeedback of physiological target response}

\section{Neurofeedback signal}

The primary concern upon receiving the feedback signal is to ensure it represents the underlying neural activity instead of physiological or movement artifact, which can contaminate BOLD measurements and thus inhibit learning self-regulation. A recent example of this was demonstrated by Zhang et al. (2011), who showed that eye movements could inflate rtfMRI training effects in those slices limited to the location of the eyes. Physiological noise from sources such as heart rate and respiration (Hu et al., 2005) and head motion (Cox and Jesmanowicz, 1999; Friston et al., 1996; Hajnal et al., 1994) are arguably the most relevant artifacts in fMRI and rtfMRI as well. Many studies have employed online motion correction and some studies measured heart rate and breathing rates as covariates for systematic errors. Tools exist to account for physiological artifacts in post-hoc analysis such as RETROICOR (Glover et al., 2000; Kasper et al., 2009), but real-time versions have yet to be published. Recent developments in signal processing in rtfMRI can further improve the robustness against such unspecific effects and noise (Hinds et al., 2011; Koush et al., 2012). One method of online physiological noise correction is to show the differential BOLD response from two different regions (Caria et al., 2007). In this way, breathing artifacts should cancel out because they can be expected to have similar effects on both target regions (Krüger and Glover, 2001). On the other hand, while some noise may be correlated, the uncorrelated Gaussian noise of the two signals will be additive, thus reducing the signalto-noise ratio.

The subject's ability to discriminate the signal from noise is likely a critical factor in the ability to self-regulate. In addition, discriminability may be affected by sensory acuity of the feedback signal. Prior to launching an rtfMRI training experiment, subjects may be tested to determine how well they can reliably detect small differences in a pre-defined, simulated biological signal designed to mimic true fMRI data that may be presented during in-scanner training ("rtfMRI psychophysics" presented by deCharms). For example, one could use any psychophysical test where the signal is initially detectable by the subject (ensuring proper sensory acuity), and then gradually increase noise. However, while such conscious discriminability of signal may facilitate learning, it may not be necessary nor sufficient for learning (Black et al., 1977).

In addition to discriminability, the temporal delay can affect the ability to self-regulate. In the great majority of studies, feedback has been continuously presented with minimal delay, approximately every $2 \mathrm{~s}$ depending on the volume acquisition rate. This may be based on methods developed using other forms of physiological feedback, such as EEG neurofeedback studies (e.g. Kotchoubey et al., 2001). As noted later in the Instructions section, subjects are typically informed of the delay prior to the experiment. Alternatively, some studies have averaged the biological signal over a longer period to achieve more meaningful information, with feedback presented after longer blocks, up to one minute in length (Bray et al., 2007; Posse et al., 2003; Shibata et al., 2011; Yoo and Jolesz, 2002). One pilot study reported improved learning in intermittent feedback compared to continuous feedback (Johnson et al., 2012). The What are the factors that influence learning? section discusses the reasoning behind this in more detail.

\section{Instructions}

Instructions to the participants is an important part of neurofeedback training (Birbaumer et al., 2008). To further reduce physiological artifacts and encourage learning, volunteers are often instructed to minimize head motion and irregular breathing in order to minimize a systematic influence of physiological artifacts on the feedback signal. It may also be explained that the signal is relatively noisy in order to manage the subject's expectations of perfect control. Typically the feedback signal and its delay with respect to neuronal activity are explained to the participants. Some studies also did short pre-trainings with computer-aided programs to acquaint participants with the delay of the feedback due to the hemodynamic response and the computing time for the feedback signal, and to ensure through task performance prior to scanning that subjects fully understand how to control the physiological information that they will see (presented by Hollman and deCharms).

A topic of debate at the conference was the primacy of implicit or explicit mental imagery strategies for self-regulation. Explicit strategies entail informing the subject of a specific means for self-regulation, whereas implicit approaches provide no such instruction and allow the subject greater room to explore different strategies. Earlier conventional wisdom suggested that providing a well-known explicit strategy to the subject would enable more efficient self-regulation, and that taking the time to find one's own effective strategy would take too long in the harsh and expensive MR environment. One unpublished study that directly compared the effects of explicit vs. potential implicit learning examined neurofeedback of the language area in 16 participants first using implicit strategies (presented by deCharms). They found no learning, despite this being chosen as a brain region that subjects can very easily control, and might be expected to readily learn implicit control over. When subjects were then given explicit strategies, they were able to learn the task. However, recent studies using implicit strategies have challenged conventional wisdom (Kim et al., 2011; Shibata et al., 2011) with one of these studies indicating learning within a single session (Shibata et al., 2011). Indeed, implicit strategies may be more advantageous since compliance to a suggested cognitive strategy cannot be quantitatively confirmed, and it may be difficult for some people to understand or report. There are also many other considerations to account for in this unresolved debate, such as the fact that some regions may have no associated explicit strategy, costly and limited scanner time, and the specific hypothesis to be tested. It may be that until the mechanisms behind such learning are better understood (see Learning mechanisms section), it may be difficult to reach a conclusion.

\section{Task design}

Most studies employ a block design for the regulation task. In this type of design, volunteers are required to regulate the BOLD signal for usually $15-30 \mathrm{~s}$ followed by a rest block of similar duration. Unlike event-related designs, block designs are less sensitive to undesired delays due to the required task switching and slow BOLD response. A single run consists of 3-6 blocks, lasts ca. 5-15 min and is repeated 2-5 times within an experimental session. The number of sessions varies significantly between studies from a single session to up to 10 sessions (Shibata et al., 2011), but the majority consisted of a single session (e.g. Caria et al., 2007; deCharms et al., 2005). Offline mental training between sessions could be advantageous towards accelerating learning (Subramanian et al., 2011), but make it difficult to separate its effect from neurofeedback training. When explicit strategies are suggested, anecdotal evidence was offered that offline coaching by the experimenters could also have a positive effect on motivation and performance (presentation by deCharms). The maximal number of runs seems to be limited by the attention span and exhaustion of volunteers, possibly affected by the type of feedback provided.

Feedback is typically presented visually as a 'thermometer display' or continuous scrolling curve representing brain activation. However, feedback has also been implemented via virtual reality, such as reaching 
for a coffee mug (Sitaram et al., 2005), computer games (Goebel et al., 2004), or a virtual fire (deCharms et al., 2005). Auditory feedback is an option, but has rarely been attempted (Posse et al., 2003). During the meeting, the impact of neurofeedback interfaces and how to potentially evaluate and optimize their design was noted as a current research gap in the field. In addition, the conference participants discussed a frequent lack of methodological detail in articles, making it difficult for other groups to replicate studies using the same processing parameters and rules for feedback display updates (i.e. methods for calculating percent signal change and descriptions of how measured changes are related to number of units and visual field angles incremented or decremented from a thermometer).

Although the majority of studies use the same training duration for all subjects in the study, it has been recognized that designs that select different amounts of training for different subjects may be more appropriate, since the individual learning curves can vary significantly. Thus, at least one study introduced individual criteria for finalizing the training (Scharnowski et al., 2012), e.g., based on achieved success of regulation in transfer trials (see Replication section). This may help to make group effects more homogeneous in following behavioral tests.

Typically, neurofeedback is conducted without any explicit sensory stimulation other than the feedback signal itself, although this need not be the case. For instance, deCharms and colleagues have trained subjects to control brain activation in response to painful stimuli (deCharms et al., 2005). Veit et al. (2012) trained participants to volitionally up- and down-regulate the anterior insula in the presence of threat-related stimuli. Another study employed down-regulation of amygdala during emotional stimuli (presented by Bruehl). Yet another study trained individuals to up- and down-regulate, in separate sessions, brain regions involved in the visual perception of emotion, when subjects were concurrently stimulated by a backward priming paradigm (Kim et al., 2011, presentation by Sitaram). The choice of stimulus-based self-regulation depends on the function to be regulated and/or the question at hand.

\section{Transfer after successful training}

Since many studies are interested in investigating potential behavioral effects learned through rtfMRI-based training that remain after the training has ended, it is important to determine whether participants are able to demonstrate enhanced brain activation or other behavioral effects when they are no longer viewing rtfMRI information, and outside the scanner. In particular, in clinical applications an important goal will be to maintain skills practiced and acquired during rtfMRI sessions and be able to apply them to real-life situations. Many studies included transfer runs that followed the same experimental design as training runs but lacked the feedback signal (e.g. deCharms et al., 2004, 2005; Ruiz et al., 2013; Sulzer et al., 2013). Usually transfer runs are conducted at the end of an experimental session or after a number of sessions. As transfer should demonstrate the degree to which the learned regulatory ability can be translated to the world outside the scanner, some studies use similar, but different paradigms compared to the training task (Caria et al., 2007). Debriefings following transfer sessions have used subjective reports of regulation success to assess placebo effects and awareness-similar to EEG feedback studies (Kotchoubey et al., 2001). More clinically-oriented studies will likely desire long-term monitoring of behavioral consequences, adding a follow-up behavioral evaluation long after rtfMRI training (see Replication section).

\section{Experimental control conditions}

The experimental controls employed in rtfMRI neurofeedback can serve various purposes. In most cases control groups have been used to determine whether the feedback signal is necessary for learning to regulate brain activation, compared to the effects on brain activation produced through repetitive training using simple instructions alone. Control groups received sham feedback that was derived from other participants' data or artificially created (Caria et al., 2010; deCharms et al., 2004, 2005; Rota et al., 2009). In other studies control groups received contingent feedback (i.e. directly related to the feedback signal), but from areas other than the experimental target region (deCharms et al., 2005; Scharnowski et al., 2012), which can control for psychoeducative (i.e. benefit from learning) effects. In another study, feedback to the control group was inverted unbeknownst to the participants, thereby encouraging down-regulation of the selected ROI to help delineate the influence of neurofeedback on self-regulation (Sulzer et al., 2013). In the sham feedback paradigm the success rates can be well-matched between the experimental and control groups, but it may not present a realistic feedback with respect to noise and contingency. However, a subject may consciously or unconsciously interpret the less representative sham or control region feedback, thereby discouraging performance and creating a placebo expectancy effect (Stroebel and Glueck, 1973). In some studies, regulation without the feedback has been used as a control condition (e.g. deCharms et al., 2005). These control conditions are all examples of negative controls, i.e. conditions that one would expect to show worse performance than the experimental group.

Positive controls represent an important future area for research, allowing the direct comparison of results achievable through rtfMRIbased training with results that can be achieved through other means. For instance, the level of activation in a target ROI produced following neurofeedback should be directly and quantitatively compared to the level of activation produced using the best-known method of exciting that region, not just with a baseline condition or a negative control (described by deCharms). This was the strategy used by Berman et al. who examined self-regulation of primary motor cortex, finding that self-regulation using finger tapping exhibited, as expected, far superior performance to that of mental imagery strategies (Berman et al., 2011). Given its cost and complexity, if rtfMRI-based training is to have practical applications, it is important to demonstrate that training can produce novel task strategies that in turn produce novel levels of activation meaningfully superior to those that can be achieved using 'best-in-class' conventional task strategies. Inclusion of positive controls is a necessary step in moving the field towards clinical translation, as it has been with other neural stimulation methods such as pharmacology, TMS, and electrical stimulation.

\section{Behavioral effects of neurofeedback}

An exciting and emerging focus for many groups using rtfMRI neurofeedback has moved from learning regulation to testing specific behavioral effects. Some examples of behavioral outcomes in previous studies include measures of finger tapping frequency (Subramanian et al., 2011), visual acuity (Scharnowski et al., 2012), and emotion recognition (Ruiz et al., 2013), amongst others. Finding changes in these outcomes is not enough to claim a benefit without proper controls (deCharms et al., 2005). Particularly in clinical studies, new possibilities and issues in controls arise aside from those mentioned in the previous section. In these studies, control groups who receive a completely different type of treatment were introduced to control for placebo effects and estimate the relative efficiency, since it may be less important to estimate the precise effect of the feedback. For example, in a study on chronic pain, rtfMRI neurofeedback was compared to skin conductance response feedback (deCharms et al., 2005). In Parkinson's disease, neurofeedback was compared to motor imagery (Subramanian et al., 2011). Within subject controls are also possible by training two mutually exclusive physiological responses. An example is the bidirectional regulation of the BOLD response. For example, in such a bidirectional control design, significantly different memory encoding effects were shown for the up- vs. down-regulation condition (Weiskopf et 
al., 2004b). Such an internal control reins in on unspecific attention and regulation effects and does not require matching of different groups. Using a different strategy, Shibata and colleagues trained each subject on one of three different grating patterns and found differential improvements based on a functionally localized ROI (Shibata et al., 2011). Placebo effects can also be controlled for by subjective reports as shown in EEG-feedback (Kotchoubey et al., 2001; Schwartz and Andrasik, 2003) but this has not yet been implemented in rtfMRI feedback.

In summary, there is currently no single "correct" experimental design in rtfMRI neurofeedback, but there are important principles that the field is rapidly developing to make experiments more likely to succeed, and their results more meaningful. While there are many basic elements that rtfMRI neurofeedback experiments have in common, experimental designs will vary depending on the specific hypothesis, ROI, behavior, and type of subject. As with most experiments, pilot testing is required to fine-tune various parameters, and to maximize learning and robustness. There still remain many fundamental open questions regarding optimization of designs, as noted in Box 1.

\section{Scientific applications}

Neurofeedback as a scientific tool was pioneered by a number of researchers in the late 1960's (Fetz, 1969; Fox and Rudell, 1968; Olds, 1965; Wyrwicka and Sterman, 1968), using electrophysiological recordings in animals either noninvasively (EEG) or invasively. These research lines continue into the present time (Jackson et al., 2006; Moritz et al., 2008; Schafer and Moore, 2011). In humans, a number of studies have demonstrated the feasibility of learning to control local brain activity using rtfMRI neurofeedback. Some of these studies have shown that learned control of brain activity leads to behavioral effects that are specific to the functional role of the targeted brain area (Bray et al., 2007; Caria et al., 2007; deCharms et al., 2005; Haller et al., 2010; Rota et al., 2009; Scharnowski et al., 2012; Shibata et al., 2011; Subramanian et al., 2011; Weiskopf et al., 2003). Whereas conventional neuroimaging measures changes in BOLD signal that are due to sensory stimulation or performing a behavioral task, neurofeedback offers the opportunity to examine how changes in BOLD signal precede changes in behavior. In other words, while in conventional neuroimaging experiments, behavioral task or sensory stimulation is the independent variable (and brain activity the dependent variable), brain activity is the independent variable in neurofeedback experiments. Similar to other interventional techniques such as transcranial magnetic stimulation (TMS), deep brain stimulation, cortical cooling, psychopharmacology, or focal lesions in patients, neurofeedback thus allows for establishing a causal link between brain activity and behavior. This section discusses the current use and potential of neurofeedback in scientific investigation through

Box 1

Open questions for study design.

1. What is the optimal study design (i.e. run length, block length, etc.) for learning and how does it vary with region and strategy?

2. When should instructed strategies be implicit or explicit?

3. Are visual feedback channels always more advantageous than other sensory channels?

4. How does feedback and the amount of information it provides affect motivation, attention and learning?

5. When is intermittent feedback more advantageous than continuous feedback? examples of neuromodulation by the individuals themselves (i.e. endogenously), and by an external source (i.e. exogenously).

The pioneering studies in neurofeedback examined how endogenous manipulation of brain activity within a single ROI affected behavior. For example, the study by deCharms et al. examined how self-regulation of rACC correlated with pain perception (deCharms et al., 2005). Instead of trying to dose-match the self-regulation as with brain stimulation, the authors correlated the ability to selfregulate rACC with reduced pain perception. The experiment also used three control groups experiencing either no feedback, yoked (i.e. from another participant) sham feedback, or feedback from a different region, none of which showed the same effects as contingent rACC neurofeedback. These controls ensured that the effect did not arise from the explicit mental strategy given, observing rACC activity, or the ability to self-regulate any region, respectively. While a strong case can be made that rACC modulates pain perception from this study, the authors admit that it is possible that rACC activity changes may be driven by top-down connections from a higher order region that causally affects both rACC activity and pain perception as independent quantities. Secondly, it may also be possible that the participants' abilities to self-regulate rACC may not be independent of the abilities to self-regulate pain. Other studies have applied similar ROI-based neurofeedback approaches to fields such as motor function by training on a ROI in the precentral gyrus (Bray et al., 2007), language by training a ROI in the inferior frontal gyrus (Rota et al., 2009), emotions by training a ROI in the insula (Caria et al., 2010), memory by training a ROI in the parahippocampal complex (Weiskopf et al., 2004b), and perception by training a ROI in the occipital cortex (Scharnowski et al., 2012). The variety of functional brain areas that have been successfully targeted with neurofeedback indicates that this approach is a versatile tool for scientific investigation whose limitations are still unknown.

Yet rtfMRI has potential beyond single ROI neurofeedback, as developments in multivariate methods have led to a new set of possibilities. There have been a number of multivariate approaches such as functional connectivity-based (presentation by Zilverstand), multiple ROIs (Chiew et al., 2012), and machine learning classifiers (LaConte et al., 2007; Sitaram et al., 2011). One study by Shibata et al. (2011) used a decoder to identify voxels in early visual cortex (V1/V2) corresponding to three different Gabor patch gratings differing by $60^{\circ}$ orientation from each other. The feedback signal communicated the likelihood of these voxels representing one of the patches, unbeknownst to the participant. Following neurofeedback training, participants improved perceptual sensitivity to the target grating compared to the other two. These different gratings were an inventive way to establish control conditions separating the ability to self-regulate from behavioral effects. To account for specificity, the authors compared activity in other related regions offline to V1/V2 activity, showing that no other connected regions could account for this change. While this study limits the ROI selection to visual cortex, the neurofeedback approach benefits from whole brain coverage and thus allows for modulating any choice of ROI(s), especially when multivariate methods are used. This represents a significant advantage over other neuromodulation techniques relegated to single cortical areas.

Recently, online measurement of brain states using rtfMRI has been used to control exogenous events, a novel approach for neuroscientific investigation. In contrast to endogenous neurofeedback, in which participants self-regulate brain activity via a feedback signal, in exogenous neurofeedback, the feedback signal is not presented to subjects, but it is instead used to trigger external stimuli in order to manipulate brain activity and/or behavior. In other words, a key difference between endogenous and exogenous neurofeedback is that in the former, the participant is aware of the feedback signal. For example, in an innovative study, Yoo and colleagues monitored the activation in a memory-related ROI in real-time and triggered a 
memory probe when participants entered "good" and "bad" brain states for learning novel scenes (Yoo et al., 2012). They found that when scenes were triggered by good ROI states, they were remembered significantly better than scenes that were triggered by bad ROI states. Hence, the activation patterns in the ROI were correlated with memory performance. However, from a causal perspective, the possibility of a higher order region or network being primarily responsible for this effect is not clear. Another study by the same group used triggering to investigate how vigilance changes with increasing activity in supplementary motor area (SMA) activity compared to increasing activity in default-mode network (DMN) regions (Hinds et al., 2013). They found increased vigilance when triggering during higher SMA activity compared to DMN activity, implicating the differential roles of the SMA and DMN, as well as a method for controlling human vigilance.

Aside from triggering a probe depending on the brain state, a largely unexplored area of rtfMRI is what is known as a "closedloop" paradigm, which exogenously manipulates brain activity. In such a paradigm, the sensory stimulation is modified depending on the current level of brain activity. For example, Gantner and colleagues changed the transparency of an image of a house depending on the level of activity in a house processing brain area (Gantner et al., 2010). The participants in this experiment were not aware that the visual stimulation was linked to their own brain activity. Instead of a brain-machine interface, such a closed loop paradigm represents a "machine-brain" interface, where the machine stimulates a particular pattern of brain activity, rather than the traditional opposite direction. However, while reducing the role of human cognition in the control loop, the challenges of a machine-brain interface migrate from mental strategies to the design of the machine controller, or in other words, the burden of how to regulate the BOLD signal relies on artificial intelligence and the availability of the appropriate stimuli. Nevertheless, this paradigm can be used to investigate neuronal dynamics with the unique advantages of repeatable and controllable manipulation over arbitrary regions within the whole brain.

These examples illustrate that rtfMRI-based paradigms are complementary to, and significantly extend the possibilities of conventional neuroimaging methods. As discussed above, single ROI-based endogenous neurofeedback can be used to establish links between specific brain regions and behavior, while more recent multivariate approaches have the advantage of taking a whole-brain network perspective of these relations. Exogenous methods such as triggering can be used to probe certain brain states for a specific online behavioral response, whereas a "machine-brain" interface can potentially selectively alter brain states within the realm of available stimuli. Taken together, similar to other interventional techniques, rtfMRI-based paradigms might allow researchers to address questions of causality rather than mere correlations between brain activity and mental functions. Compared to the other interventional techniques, the main advantage of rtfMRI-based paradigms is that they are noninvasive (compared to deep brain stimulation, cortical cooling, or psychopharmacology), that they allow for whole brain coverage, even of subcortical structures (compared to TMS), that they allow for a high spatial resolution (compared to TMS and psychopharmacology), and that they allow for a flexible choice of the target ROI (compared to brain lesions, psychopharmacology, and TMS). Another advantage of the neurofeedback approach is that the learning as well as executing the learned control over brain activity takes place in the MR scanner. This allows for investigating plastic changes that take place due to neurofeedback training as well as for investigating how modulating brain activity in the trained ROI affects processing in other brain areas (Lee et al., 2011, 2012; Rota et al., 2011). At this point, we have not been able to identify any topics in the cognitive neurosciences addressed with fMRI that cannot also be investigated with rtfMRI-based approaches. Given the novelty of the field, important questions regarding its use are beginning to be addressed, examples of which are provided in Box 2.

\section{Clinical applications}

Disorders of the brain, ranging from stroke to addiction to autism, represent one of the crucial public health challenges for rtfMRI neurofeedback. The following section describes the steps to be taken and risks to be considered if neurofeedback is to play a role in addressing this challenge.

Although a large variety of brain disorders could be imagined in principle as targets for neurofeedback, robust and well-controlled studies on patients based on well-founded pathophysiological models must lead the way. Until now, studies using rtfMRI neurofeedback have shown that healthy subjects can self-regulate a number of different brain regions during scanning, and a smaller number of studies have investigated patient populations. Less is known about the ability of patients with neurological and psychiatric disorders to learn self-regulation of focal brain activity through rtfMRI neurofeedback and the behavioral effects thereof. Previous literature in EEG neurofeedback has shown the ability to self-regulate brain activity in patients suffering from psychiatric and neurological disorders, including ADHD and epilepsy (Birbaumer et al., 2008). To date, several individual pilot studies in rtfMRI neurofeedback have reported training success with different patient groups (Table 1 ).

\section{Which neural circuit to train?}

One methodological aspect in the development of therapeutic rtfMRI neurofeedback is to differentiate between training aimed at improving deficient neural circuitries directly versus training "compensatory" circuits to take over for lost or impaired function. The success of training aimed at deficient circuits, which is akin to the approach generally taken in the development of deep-brain stimulation protocols, depends on sound knowledge of these circuits, for example when the model of a disorder is well-supported by multimodal evidence (animal studies, human studies, stimulation/lesion studies). The ROI or network targeted by rtfMRI neurofeedback should be accurately represented based on neuroscientific and clinical knowledge of the pathophysiology of the disorder at hand, which is a particular challenge in those psychiatric disorders where no clinically suitable imaging biomarkers have been identified (Linden, 2012a).

If the specific biological mechanism of a disease state or other impairment is not well-known, an alternative would be to target potentially compensatory networks that have been well studied in the healthy population. One example of relevance to psychiatry is the putative networks for automatic and voluntary emotion regulation (Ochsner et al., 2012; Phillips et al., 2008), which provide multiple targets for region- or network-based neurofeedback training (Esmail and Linden, 2011; LaConte et al., 2007; Sitaram et al., 2011). If we assume that patients have a clinical, psychological or cognitive deficit in a particular

\section{Box 2}

Open questions for scientific applications.

1. What are the limitations of rtfMRI for scientific investigation in cognitive neuroscience?

2. How can the specific advantages of endogenous and exogenous methods be used for scientific investigation?

3. Can it be proven that excitation, endogenously or exogenously elicited, truly causes an action?

4. How can introspective measures related to mental strategies be quantified/classified? 
Table 1

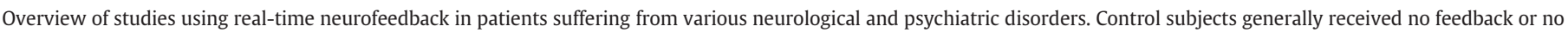
real feedback ("sham-feedback").

\begin{tabular}{|c|c|c|c|}
\hline Study & Disorder & $\mathrm{N}$ subjects/control group & Brain regions \\
\hline deCharms et al. (2005) & Chronic pain & $12 / 36^{\mathrm{a}}$ & ACC \\
\hline Ruiz et al. (2013) & Schizophrenia & $9 / 0$ & Insular cortex \\
\hline Haller et al. (2010) & Chronic tinnitus & $6 / 0$ & Auditory cortex \\
\hline Subramanian et al. (2011) & Parkinson's disease & $5 / 5$ & Supplementary motor complex \\
\hline Linden et al. (2012) & Major depression & $8 / 8$ & $\begin{array}{l}\text { Brain regions involved in positive emotions (VLPFC R/L, insular cortex R/L, } \\
\text { DLPFC R/L, medial temporal lobe } R / L, O F C \text { ) }\end{array}$ \\
\hline Sitaram et al. (2012) & Chronic stroke & $2 / 4^{\mathrm{b}}$ & Ventral premotor cortex L \\
\hline Li et al. (2012) & Nicotine addiction & $10 / 0$ & $\mathrm{ACC}, \mathrm{mPFC}$ \\
\hline
\end{tabular}

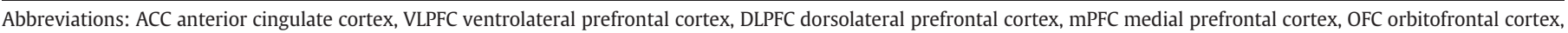
$\mathrm{R}$ right, $\mathrm{L}$ left.

a Healthy subjects as control participants, furthermore other groups receiving different forms of feedback and training (4 patients, 24 healthy subjects).

b Healthy subjects as control group.

domain, for example in emotion regulation, it might make sense to engage them in a training process involving the relevant circuits without requiring demonstration of a primary deficit in these circuits. Although it may sound unsatisfactory to apply a treatment protocol without first demonstrating a biological deficit, this approach has been successfully implemented in nearly all psychiatric (both biological and psychological) therapeutics (Linden, 2012a). However, one potential problem with this approach is that functional networks in patients may differ from those in the healthy population as a result of primary deficits or adaptations to the disease process. This problem can be addressed by identifying target areas through individual functional localizer scans. For example, a recent study on depression by some of the co-authors identified the brain areas responsive to positive emotional stimuli at the start of each neurofeedback session and used these functionally defined areas as ROIs for the self-regulation training (Linden et al., 2012).

\section{Potential risks}

One of the first steps before clinical implementation is to evaluate the potential risks involved. When safety guidelines are properly followed, MRI and fMRI are regarded as relatively risk-free methods. In over 20 years of application, no severe adverse events have occurred or side effects have been detected as long as safety guidelines are followed (Bourland et al., 1999; Schaefer et al., 2000; Schenck, 2000; Shellock and Crues, 2004). A recent safety study that directly investigated potential adverse events related to fMRI and related to rtfMRIbased training has demonstrated that neither fMRI nor rtfMRI-based training produced adverse events at greater rates than those associated with non-scanning controls, even in chronic pain patients who are particularly susceptible to side effects (Hawkinson et al., 2012). However, one study in schizophrenic patients found that they detected disgust faces better with up-regulation of anterior insula (Ruiz et al., 2013). A potential risk is that maladaptive neural plasticity could be induced, for instance, by repeated rtfMRI neurofeedback training of dysfunctional strategies. The only side effects commonly observed to date include mental fatigue and physical discomfort, natural accompaniments to experiments that require concentration and minimal head movement in the scanner. In addition, people may also feel claustrophobic in such a tight space, which limits the potential patient population, and the scanner is loud which can lead to ringing in the ears.

\section{Determining effect size}

After showing the general applicability of rtfMRI neurofeedback for training of regulatory abilities in mental disorders, the method needs to undergo scrutiny by the methods of evidence-based medicine. First, one must determine the effects of rtfMRI neurofeedback in healthy participants (e.g. Bray et al., 2007; Shibata et al., 2011; Weiskopf et al., 2003). Typically, the next step would be "dose-finding" protocols on small patient groups to determine the maximum tolerated and the clinically meaningful or necessary dosage. However, it remains an interesting open question as to whether patients are even capable of reaching such maximum levels of neurofeedback performance, or even if it is possible to achieve at all using endogenous means. While trials on small patient groups using rtfMRI training have been conducted (see Table 1), none have witnessed adverse effects from excessive selfregulation, as discussed in the Potential risks section. Finally, it should be noted that no studies using rtfMRI training have yet conducted follow-up examinations, such as after 3, 6 and 12 months, despite their importance in determining its effect (e.g. Craske et al., 1991; Öst and Westling, 1995).

\section{Randomized controlled trials and multicenter studies}

In further stages of investigation, rtfMRI neurofeedback will have to prove its clinical utility in comparison with alternative therapeutic methods, for example psychotherapy, pharmacotherapy, physiotherapy or other physical interventions. This will require randomized clinical trials. These studies will address the efficacy and generalizability of neurofeedback while revealing risks and side effects in comparison to other methods (Jacobson and Christensen, 1996), such as biofeedback using peripheral mechanisms and experimenter guidance, likely in a multi-center design. The challenges of these clinical trials include high numbers of subjects (depending on effect size), well-defined controlgroups such as sham feedback and alternative stimulation methods, and close communication between participating centers to ensure data stability and consistency (e.g. multicenter studies on the efficacy and mechanisms of psychotherapy as conducted by Gloster et al., 2009).

\section{Replication}

Ensuring reproducibility is a key challenge in neurofeedback studies. One of the landmark works in the field found self-regulation of rACC could reduce pain scores in chronic pain patients compared to controls in a single session (deCharms et al., 2005). A follow-up trial consisted of six training sessions, approximately weekly, and using six different explicit mental strategies, on a larger number of subjects (21 experimental, 38 sham). The cognitive strategies used by all of the subjects to control their pain had been derived and optimized during prior rtfMRI-based training sessions. These experiments, presented by deCharms, showed that the subjects' pain symptoms improved markedly. In addition, subjects who received rtfMRI-based training during the experiment improved their ability to control brain activation in target ROIs related to the pain system, while control subjects who received identical training but sham rtfMRI information had no improvements in 
their control over brain activation. However, on the symptoms level, the two groups had very similar changes in their pain ratings-both groups improved similarly. This suggests two important possibilities: 1) it may be that clinically meaningful improvements in patients can be achieved using strategies derived previously from rtfMRI-based training experiments, even in the absence of scanning; 2 ) it may be, in this application, that rtfMRI-based training itself does not produce greater benefit to patients than the benefit produced by appropriately-selected strategies.

Caution against overstating progress should be exercised. Despite the proof-of-principle in healthy subjects and preliminary results in some patient groups, a real usefulness in clinical routine is far from being demonstrated (see open questions listed in Box 3). The reader should be cautioned that projecting practical clinical results from rtfMRI remains a future goal; currently, even the more basic routine diagnostic use of fMRI has yet to be established in wide clinical practice. Nevertheless, the growing interest in fMRI-based neurofeedback and its clinical applications is likely to also lead to a deeper understanding of the brain processes underlying neurological and psychiatric disorders, and potentially to the development of novel forms of treatment.

\section{Learning mechanisms}

While there are several studies demonstrating rtfMRI as a scientific tool or a therapeutic method, there are very few studies targeted at testing specific theoretical hypotheses about the mechanism of operant and cognitive control of neural activity with feedback. Gaining an understanding of and then exploiting these learning mechanisms could help standardize and quantify methods used in the field. In this chapter, we discuss some fundamental questions raised in the conference regarding what model best represents neurofeedback-associated learning mechanisms.

\section{What are the learning mechanisms engaged by neurofeedback?}

Learning via rtfMRI training entails a number of possible learning mechanisms. This section discusses two overarching concepts, namely, associative learning and information representation in light of rtfMRI neurofeedback training.

Associative learning is the process by which an association between two stimuli, or between a behavior and a stimulus is learned. The two forms of associative learning are classical and operant conditioning. Operant learning theory, often used to explain neurofeedback learning, states that the probability of a physiological response is increased when a reinforcing stimulus follows that response. The theory focuses on three main elements: (1) discriminative stimuli (SDs), (2) responses, and (3) reinforcers. When the response is reinforced in

\section{Box 3}

Open questions for clinical applications.

1. In which neurological diseases is rtfMRI neurofeedback appropriate, and under what conditions is it inappropriate?

2. Under which conditions is rtfMRI neurofeedback more advantageous than other interventions?

3. To what extent is the behavior of healthy participants a model for patients?

4. Can self-regulation be repeated outside the clinic?

5. How effective is the treatment, and how long does the effect last?

6. What are the side-effects?

7. Is there a maximum dosage a patient can provide oneself? the presence of one SD (e.g. a visual symbol of an up-arrow) and not in the presence of other SDs (e.g. visual symbol of a down-arrow), the increase in response probability will occur only in the presence of the first SD. In the case of a rtfMRI neurofeedback experiment, a reinforcing stimulus could be the real-time feedback of the brain activity, for example, in the form of increase in the bars of a thermometer in proportion to the amplitude of the BOLD signal in a brain region relative to baseline or a given reference activity.

In recent review papers on learning and memory (Dickinson, 2012; Gallistel and Matzel, 2013), prominent neuroscientists in the field discussed two major extant theoretical frameworks, namely, associative learning and information representation. According to the current view, associative learning forms input-output mappings between stimulus and response without representing the environment that makes the behavior adaptive. In contrast, most contemporary cognitive neuroscience theories of learning and memory are representational; learning mechanisms extract useful information from experience, while memory carries the acquired information forward in time in a computational form that could be retrieved and used by the subject (Gallistel and King, 2009). Representational and higher-order cognitive theories are centered on the concept of expectancy, defined as a future-oriented belief, as more than the activation of simple binary associations. In higher organisms like humans, behavioral flexibility requires greater complexity, forming associations via conditioning procedures as well as from other sources of information. Recently, Koralek et al. demonstrated that controlling brain activity is a form of abstract skill by using a novel paradigm of auditory cursor control in rodents trained to modulate the electrophysiological signals of the primary motor cortex (Koralek et al., 2012). Remarkably, the authors showed the specific involvement of cortico-striatal plasticity by deletion of NMDA receptors which impaired this skill. In light of these results and earlier studies that show that learning an abstract skill involves cognitive or representational aspects (Beauchamp et al., 2003), we can surmise that learning may involve both methods discussed above, with the greater involvement of information representation in the learning process with greater complexity of self-regulation, a hypothesis that could be investigated with future rtfMRI studies (Dayan and Cohen, 2011).

\section{What are the psychobiological mechanisms of neurofeedback?}

The physiological basis of learning is still evolving. The neurobiological process believed to be the basis of associative learning is long-term potentiation (LTP). Current opinion emphasizes the role of a specific form of LTP called spike timing-dependent plasticity (Caporale and Dan, 2008), although recently this established theory has been questioned (Gallistel and Matzel, 2013).

While much is known regarding how BOLD changes are associated with underlying neural changes, a complete understanding of neurovascular coupling is still not clear. It is known that both neural excitatory and inhibitory responses lead to increases in the BOLD signal (Logothetis, 2008). Given this, we cannot say whether voluntary up-regulation of BOLD leads in summary to neural excitation or inhibition. The resulting behavioral effects do shed a light on this issue. However, one might assume that induced neuronal activation would lead to consolidation or strengthening of the used connections and networks, perhaps through Hebbian mechanisms of learning (Hebb, 1949; Martin and Morris, 2002). Another potential mechanism, although perhaps less likely, is that modulation of the vascular activity is learned operantly which then changes BOLD as a consequence (Moore and Cao, 2008). Future animal and human studies of rtfMRI self-regulation, conducted during combined BOLD and electrophysiological measurements, might help answer the above questions, not only throwing further light on the brain mechanisms of volitional regulation, but also on neurovascular coupling. 
Are there limits to operant learning and how can they be overcome?

It has been shown that BCIs based on operant learning of electrical or hemodynamic brain responses can be used by paralyzed people to select letters or words with their EEG recorded brain activity and thus restore communication (Birbaumer, 2006; Birbaumer and Cohen, 2007; Birbaumer et al., 2008; Buch et al., 2012; Vaadia and Birbaumer, 2009). However, despite repeated efforts, it has thus far not been possible to train BCI-use in the completely locked-in state and in vegetative state (Kübler and Birbaumer, 2008; Ramos Murguialday et al., 2009). It should be noted that work is currently in progress to address this specifically with rtfMRI-based spellers (presentation by Goebel). In light of this, Birbaumer et al. have proposed that extinction of voluntary goal-directed behavior and goal-directed thinking after prolonged periods of complete lack of movement contingencies is responsible for this failure despite intact input-oriented cognitive processing (Birbaumer et al., 2012). Birbaumer pointed out that the problem of replicating operant learning of autonomic responses in the curarized rat (Miller, 1975; Miller and Dworkin, 1974) may constitute an analog to the failure of brain communication in complete paralysis. Technical alternatives to non-invasive BCIs such as communication with saliva pH-changes (Wilhelm et al., 2006), sniffing (Plotkin et al., 2010), functional near infrared spectroscopy (fNIRS; Naito et al., 2007; Sitaram et al., 2007) and invasive recordings (Hochberg et al., 2006; Ramos Murguialday et al., 2009) cannot overcome the described psychological learning deficit which may be even more profound in vegetative ("apallic") state where patients spend years in unresponsive positions despite partially intact cognition (Kotchoubey et al., 2005; Monti et al., 2010). The above problem could reflect a physiological and methodological constraint to any form of operant training including rtfMRI neurofeedback. In view of this problem, Birbaumer and colleagues have planned to experimentally test a solution to this fundamental disorder of volition and loss of communication abilities through twoprocess learning of brain-responses: first by using "reflexive" classical conditioning of brain responses, and second by the enhancement of the acquired response through instrumental conditioning (Liberati and Birbaumer, 2012; Liberati et al., 2012).

\section{What are the factors that influence learning?}

The biofeedback literature and recent findings from rtfMRI studies suggest the following major factors that affect learning: contingency, contiguity, instructed reinforcement, and shaping and chaining.

Contingency refers to the conditional probability of reinforcement given a response or given a failure to respond. The study of this factor includes the investigation of different modalities (visual, auditory, tactile, etc.) of the response-contingent stimuli, their different physical properties (such as amplitude, rate and the complexity), and the different functional relationships between the response and the feedback. In rtfMRI neurofeedback studies, the contingency of the feedback is often manipulated as a control condition, such as sham feedback (e.g. deCharms et al., 2005).

Temporal contiguity refers to the time interval between response and reinforcement. In an rtfMRI setting, the intrinsic delay between the neural activity that is regulated and the BOLD changes due to the slow hemodynamic response is known. Added to this would be the delay in acquisition and computation of the feedback signal. Johnson et al. (2012) showed that intermittent presentation of feedback (about 20 s delay) is more effective than continuous presentation when an imagery-based strategy was used for self-regulation. Intermittent feedback could be more advantageous in certain situations, such as during the early stage of learning, as it does not interfere with the ongoing imagery during self-regulation.

Methods employed for training voluntary control generally contain two procedural elements: instructions and response-contingent stimulation. Although for reasons of historical bias, the experimental analysis of voluntary control has tended to emphasize the role of feedback and has neglected the influence of instructions, data suggests that instructions are not at all neutral in influencing voluntary control (Schwartz and Beatty, 1977). Subjects tend to report what instructions lead them to expect. In view of this, the investigation of experimental instructions is relevant for the analysis of voluntary control (see Instructions section). Furthermore, psychological and behavioral tasks designed to test the effect of neurofeedback training must look for sensitive yet robust measures that control for effects of placebo and instruction. Shaping and chaining have received attention in the biofeedback literature (Black et al., 1977) but have not been explicitly elaborated and investigated in rtfMRI studies. The nature of the feedback itself can be deconstructed into components that may individually or in concert affect learning. How these parameters may be tuned and to what extent remains one of many open questions (see Box 4).

\section{Where is the future of rtfMRI neurofeedback?}

Since its introduction in 1995 (Cox et al., 1995) rtfMRI has inspired research leading towards neural intervention, intraoperative procedures, brain-computer interfaces and quality assurance. While the future of rtfMRI neurofeedback can lead towards some exciting applications in a multitude of neurological and psychiatric disorders, we are currently just beginning to scratch the surface of where it can be applied. This section discusses both the immediate future and long-term future of fMRI-based neurofeedback.

\section{Immediate future}

Naturally, the future of rtfMRI coincides with that of fMRI (for review on advances in fMRI, see Wald, 2012). Recent work has made measurement of more specific regions possible. For instance, imaging of the function of microcolumnar structures is being implemented, including using higher static fields such as 7T and high resolution grid sampling (presented by Goebel). Goebel mentioned the availability of ultrafast sequences that could allow very low TRs, improving contrast-to-noise ratio. This strategy is already being investigated by other groups (Posse et al., 2012). Another method of obtaining more specificity using multivariate pattern analysis (MVPA) was presented, distinguishing cortical representation of individual fingers in real time in the primary somatosensory cortex (presented by Kaas). Taken together, fMRI is becoming more specific and faster. However, the delay due to the hemodynamic response will remain as a constant which has to be considered in future even with faster measuring and calculations.

Hyperscanning is a technique developed to measure brain activity from more than one subject simultaneously during social interaction (Montague et al., 2002), which can be combined with neurofeedback training (Goebel et al., 2004). A successful implementation of rtfMRI

Box 4

Open questions for learning mechanisms.

1. To what extent do feedback factors such as feedback delay, contingency, reinforcement, motivation, instructions and manipulations differentially affect learning?

2. What neural correlates underlie neurofeedback learning?

3. How do explicit and implicit strategies affect learning?

4. Is the level of activation using neurofeedback that can be reached greater than that with a predefined task?

5. To what extent can the signal be discerned spatially and temporally? 
hyperscanning was presented using navigation through simple competitive and cooperative tasks through motor imagery, and a further example implementing a virtual environment to examine cooperation (presentation by Baecke). Hyperscanning, especially in a virtual environment, has potential for use in social neuroscience experiments, specifically neuroeconomics studies, human-computer interaction and human-computer-human interaction.

Magnetic resonance spectroscopy (MRS) can quantify the concentration of certain specific chemical compounds, for example GABA and creatine (Castillo et al., 1996). To date, real-time functional MRS has already been used to quantify dynamic BOLD changes in real-time (Koush et al., 2011). In the same manner as rtfMRI neurofeedback, real-time MRS could be used to manipulate neurotransmitter production or track brain metabolites (presentation by Koush).

Another alternative acquisition method to traditional fMRI is arterial spin labeling (ASL). ASL traces arterial blood as it flows into the brain (regional cerebral blood flow, rCBF) by "tagging" arterial blood magnetically and then measuring the response approximately one second later in the brain (Detre et al., 1994) and comparing it to a "non-tagged" control condition. It has poorer temporal resolution than EPI as a result but the advantages of a physiological and clinically meaningful outcome measure in rCBF and a true baseline. Real-time ASL (rtASL) has recently been reported (Hernandez-Garcia et al., 2011). Although currently the signal-to-noise ratio is not as good as EPI and there are still issues with selecting the optimal feedback region, the future for rtASL is promising due to its inherent advantages over BOLD signal related methods (presentation by Várkuti). Some potential uses of rtASL include tracking thrombolysis in ischemic stroke or anesthesia depth. Indeed, the disadvantage of the lower temporal resolution could be negated through experiments focused on brain regions that cannot be so quickly modulated, since ASL does not suffer from signal baseline drifts like BOLD imaging. However, as ASL is based on a similar neurovascular coupling reaction as BOLD imaging, the hemodynamic response with its delay is common to both methods.

Feedback of network or connectivity-related activity may better represent brain physiology than region-based methods and exploit the advantages of whole brain coverage. Some trends towards MVPA in rtfMRI were presented at the conference (presentations by LaConte and Goebel), including an experiment examining support vector machine (SVM) classification of emotional states (presentation by Rana), as well as recently published examples using sparse logistic regression (Shibata et al., 2011). Some examples of connectivity feedback included experiments using connectivity between the inferior frontal and superior temporal gyrus (presentation by Ruiz) and between bilateral motor cortices (presentation by Zilverstand). Both functional and effective connectivity methods, as well as multivariate pattern classification, could represent part of a larger movement towards multivariate feedback.

\section{The longer term future}

Predicting where such a fast-changing field will emerge in the next two decades is a difficult task. The current direction would suggest higher contrast-to-noise, more physiologically-related signals from multiple, more precise areas of the brain will be accessible. At the same time, more advanced computational methods of state classification and signal conditioning are being developed that will further improve robustness and selectivity of rtfMRI. As a result, rtfMRI protocols will likely become more varied before they begin to settle to some accepted design principles.

For therapeutic purposes, one would assume that clear physiological signals facilitate better neurofeedback performance. Subsequently, the functional consequences of such self-control will become more clearly defined, and thus more accurately identify ideal candidates.
Standardization of transfer will be established and compared with specific behavioral and psychological measures during neurofeedback. It is well imaginable that in the next decades rtfMRI neurofeedback could enter the phase of clinical treatment of specific neurological or mental disorders where invasive intervention is not appropriate. Neurofeedback could also be used as a complement with other therapeutic methods, e.g. physical rehabilitation delivered via MRcompatible robotic manipulation (Gassert et al., 2006, 2008). While training in the scanner may not be feasible on a long-term basis, the aim would be to have the patient transfer this learned ability for use ubiquitously outside the scanner. Also, it is possible that rtfMRI will be used as a method for developing and testing novel therapeutic interventions that are then used in later subjects outside of the scanner.

Apart from use of rtfMRI neurofeedback for therapy, the application of rtfMRI in psychiatry could also consist in identifying the neural correlates of certain mental or psychotherapeutic interventions in patients and then optimizing these interventions. Such online, whole-brain diagnostic procedures could help immediately evaluate the effectiveness of psychotherapeutic sessions or identify eligible patients for certain psychotherapies, specifically towards treatment response prediction. It may also serve for gaining knowledge about the neurobiological backgrounds of mental interventions applied in a psychotherapeutic context.

FMRI BCIs could be useful in applications that require precise measurement of whole brain activity. This technology could be of great use for patients unable to communicate by any other means, including EEG-based BCIs (Sorger et al., 2012). For bedside BCIs used daily, expensive, stationary and slow technology such as fMRI may not be feasible, but for situations that require relatively high spatial resolution compared to EEG, fNIRS could lead the way (presented by Zimmermann).

Perhaps the most immediate application of rtfMRI is in quality assurance (Weiskopf et al., 2007). As scanner manufacturers further implement real-time packages in their own software, clinicians will be able to ensure contrast integrity, motion parameters and identify electromagnetic interference with additional research or clinical equipment. In the future, it may be possible that scanner sequences will optimize themselves to improve contrast, and maybe even adjust for movement artifacts. The ability to compensate for movement online, perhaps using ultrafast sequences, would open up a whole new range of tests that could be conducted in the scanner.

There are still fundamental questions about rtfMRI that may need to be addressed before the technology is ready for clinical translation. Issues such as learning to control the signal with the hemodynamic delay, optimal sensory channels for feedback and feedback design, how to maximize signal-to-noise ratio, what strategies are best to facilitate learning and control, whether the effect size is clinically relevant, and whether the training can be transferred outside the scanner are critical to understand. While many studies have addressed some of these issues within a specific application, these questions should be revisited before applying rtfMRI to any new field.

\section{Conclusions from the meeting}

Over the past decade much work has shown promise for rtfMRI in neurofeedback and other applications. Some key successes, including showing relevant behavioral effects of neurofeedback, exhibiting its use as a scientific tool, and identifying online brain states have led to a recent spike in interest in the field. Yet despite clear progress, fundamental issues remain such as the minimum discernible signal-to-noise ratio of feedback, imagery strategy, effect size, transfer, and how participants learn to self-regulate their BOLD signal. Following the talks and discussions, it was agreed that these issues and current ones should be discussed every two years at this conference, open to the worldwide community. In addition, a mailing list was created to share general thoughts, problems, job announcements, or other relevant information to the field (email James Sulzer at jamessulzer@gmail.com to register). 


\section{Acknowledgments}

The authors would like to thank the sponsors of the event, including the Swiss National Science Foundation (project \#31C030_139955), the Zurich Neuroscience Center (ZNZ), Philips Medical, ETHZ, and the University of Zurich. NB is supported by the Deutsche Forschungsgemeinschaft (DFG, Koselleck Grant) and a European Research Council (ERC) grant and a Computational Neuroscience Grant (Bernstein) from the German Ministry of Education and Research (BMBF). FS is supported by the Swiss National Fund and a Marie-Curie Re-integration Grant of the European Union.

\section{Appendix A. List of presenters (alphabetical order)}

Sebastian Baecke (Otto-von-Guericke University in Magdeburg, Germany)

Niels Birbaumer (University of Tuebingen, Germany and Oespedale San Camilo, Venice, Italy)

Maria Laura Blefari (ETHZ, Switzerland)

Annette Bruehl (Zürich University Hospital for Psychiatry,

Switzerland)

Leonardo Cohen (National Institutes of Health, USA)

Christopher deCharms (Omneuron, USA)

Rainer Goebel (University of Maastricht, the Netherlands)

Sven Haller (University of Geneva, Switzerland)

Maurice Hollman (Max Planck Institute Leipzig, Germany)

Amanda Kaas (Maastricht University, the Netherlands)

Yury Koush (Aachen University, Germany)

Stephen LaConte (Virginia Tech University, USA)

David Linden (University of Bangor, UK)

Mohit Rana (University of Tuebingen, Germany)

Sergio Ruiz (University of Tuebingen, Germany)

Frank Scharnowski (University of Geneva, Switzerland)

Sigrid Sherpiet (Zürich University Hospital for Psychiatry,

Switzerland)

Ranganatha Sitaram (University of Tuebingen, Germany and

University of Florida, USA)

James Sulzer (ETHZ, Switzerland)

Bálint Várkuti (University of Tuebingen, Germany)

Nikolaus Weiskopf (University College London, UK)

Anna Zilverstand (University of Maastricht, the Netherlands)

Raphael Zimmermann (ETHZ, Switzerland)

\section{References}

Beauchamp, M., Dagher, A., Aston, J., Doyon, J., 2003. Dynamic functional changes associated with cognitive skill learning of an adapted version of the Tower of London task NeuroImage 20 (3), 1649.

Berman, B.D., Horovitz, S.G., Venkataraman, G., Hallett, M., 2011. Self-modulation of primary motor cortex activity with motor and motor imagery tasks using realtime fMRI-based neurofeedback. NeuroImage 59 (2), 917-925.

Birbaumer, N., 2006. Breaking the silence: brain-computer interfaces (BCI) for communication and motor control. Psychophysiology. 43 (6), 517-532.

Birbaumer, N., Cohen, L.G., 2007. Brain-computer interfaces: communication and restoration of movement in paralysis. The Journal of Physiology 579, 621-636.

Birbaumer, N., Murguialday, A.R., Cohen, L., 2008. Brain-computer interface in paralysis. Curr. Opin. Neurol. 21 (6), 634

Birbaumer, N., Piccione, F., Silvoni, S., Wildgruber, M., 2012. Ideomotor silence: the case of complete paralysis and brain-computer interfaces (BCI). Psychol. Res. 1-9.

Biswal, B., Zerrin Yetkin, F., Haughton, V.M., Hyde, J.S., 1995. Functional connectivity in the motor cortex of resting human brain using echo-planar MRI. Magn. Reson. Med. 34 (4), 537-541.

Black, A., Cott, A., Pavloski, R., 1977. The operant learning theory approach to biofeedback training. Biofeedback: Theory and Research, pp. 89-127.

Bourland, J.D., Nyenhuis, J.A., Schaefer, D.J., 1999. Physiologic effects of intense MR imaging gradient fields. Neuroimaging Clin. N. Am. 9 (2), 363.

Bray, S., Shimojo, S., O'Doherty, J.P., 2007. Direct instrumental conditioning of neura activity using functional magnetic resonance imaging-derived reward feedback. J. Neurosci. 27 (28), 7498-7507.

Buch, E.R., Shanechi, A.M., Fourkas, A.D., Weber, C., Birbaumer, N., Cohen, L.G., 2012. Parietofrontal integrity determines neural modulation associated with grasping imagery after stroke. Brain. 135 (2), 596-614.
Caporale, N., Dan, Y., 2008. Spike timing-dependent plasticity: a Hebbian learning rule. Annu. Rev. Neurosci. 31, 25-46.

Caria, A., Veit, R., Sitaram, R., Lotze, M., Weiskopf, N., Grodd, W., Birbaumer, N., 2007. Regulation of anterior insular cortex activity using real-time fMRI. NeuroImage 35 (3), 1238-1246.

Caria, A., Sitaram, R., Veit, R., Begliomini, C., Birbaumer, N., 2010. Volitional control of anterior insula activity modulates the response to aversive stimuli. A real-time functional magnetic resonance imaging study. Biol. Psychiatry 68 (5), 425-432.

Caria, A., Sitaram, R., Birbaumer, N., 2012. Real-time fMRI: a tool for local brain regulation. Neuroscientist 18 (5), 487-501.

Castillo, M., Kwock, L., Mukherji, S.K., 1996. Clinical applications of proton MR spectroscopy. Am. J. Neuroradiol. 17 (1), 1-15.

Chapin, H., Bagarinao, E., Mackey, S., 2012. Real-time fMRI applied to pain management. Neurosci. Lett. 520 (2), 174-181.

Chiew, M., LaConte, S.M., Graham, S.J., 2012. Investigation of fMRI neurofeedback of differential primary motor cortex activity using kinesthetic motor imagery. Neurolmage 61 (1), 21-31.

Cox, R.W., Jesmanowicz, A., 1999. Real-time 3D image registration for functional MRI. Magn. Reson. Med. 42 (6), 1014-1018.

Cox, R.W., Jesmanowicz, A., Hyde, J.S., 1995. Real-time functional magnetic resonance imaging. Magn. Reson. Med. 33 (2), 230-236.

Craske, M.G., Brown, T.A., Barlow, D.H., 1991. Behavioral treatment of panic disorder: a two-year follow-up. Behav. Ther. 22 (3), 289-304.

Dayan, E., Cohen, L.G., 2011. Neuroplasticity subserving motor skill learning. Neuron 72 (3), 443-454.

deCharms, R.C., 2007. Reading and controlling human brain activation using real-time functional magnetic resonance imaging. Trends Cogn. Sci. 11 (11), 473-481.

deCharms, R.C., 2008. Applications of real-time fMRI. Nat. Rev. Neurosci. 9 (9), 720-729.

deCharms, R.C., Christoff, K., Glover, G.H., Pauly, J.M., Whitfield, S., Gabrieli, J.D.E., 2004. Learned regulation of spatially localized brain activation using real-time fMRI. NeuroImage 21 (1), 436-443.

deCharms, R.C., Maeda, F., Glover, G.H., Ludlow, D., Pauly, J.M., Soneji, D., Gabrieli, J.D.E., Mackey, S.C., 2005. Control over brain activation and pain learned by using realtime functional MRI. Proc. Natl. Acad. Sci. U.S.A. 102 (51), 18626.

Detre, J.A., Zhang, W., Roberts, D.A., Silva, A.C., Williams, D.S., Grandis, D.J., Koretsky, A.P., Leigh, J.S., 1994. Tissue specific perfusion imaging using arterial spin labeling. NMR Biomed. 7 (1-2), 75-82.

Dickinson, A., 2012. Associative learning and animal cognition. Philos. Trans. R. Soc. Lond. B Biol. Sci. 367 (1603), 2733-2742.

Elbert, T., Rockstroh, B., Lutzenberger, W., Birbaumer, N., 1980. Biofeedback of slow cortical potentials. I. Electroencephalogr. Clin. Neurophysiol. 48 (3), 293-301.

Esmail, S., Linden, D.E.J., 2011. Emotion Regulation Networks and Neurofeedback in Depression. Cognitive Sciences. 6 (2).

Frank, S., Lee, S., Preissl, H., Schultes, B., Birbaumer, N., Veit, R., 2012. The Obese Brain Athlete: Self-Regulation of the Anterior Insula in Adiposity. PloS One. 7 (8), e42570.

Feinberg, D.A., Moeller, S., Smith, S.M., Auerbach, E., Ramanna, S., Glasser, M.F., Miller, K.L., Ugurbil, K., Yacoub, E., 2010. Multiplexed echo planar imaging for subsecond whole brain FMRI and fast diffusion imaging. PLoS One 5 (12), e15710.

Fetz, E.E., 1969. Operant conditioning of cortical unit activity. Science 163 (3870), 955-958.

Fox, S.S., Rudell, A.P., 1968. Operant controlled neural event: formal and systematic approach to electrical coding of behavior in brain. Science 162 (3859), 1299.

Friston, K.J., Williams, S., Howard, R., Frackowiak, R.S.J., Turner, R., 1996. Movementrelated effects in fMRI time-series. Magn. Reson. Med. 35 (3), 346-355.

Gallistel, C.R., King, A.P., 2009. Memory and the Computational Brain: Why Cognitive Science Will Transform Neuroscience. Wiley-Blackwell.

Gallistel, C., Matzel, L.D., 2013. The neuroscience of learning: beyond the Hebbian synapse. Annu. Rev. Psychol. 64, 169-200.

Gantner, I.S., Hutton, C., Scharnowski, F., Kaas, A., Weiskopf, N., 2010. Unconscious Closed-loop Feedback to the Visual System for Quantifying the Effectiveness of BOLD-based Neurofeedback. Amsterdam, The Netherlands.

Gassert, R., Moser, R., Burdet, E., Bleuler, H., 2006. MRI/fMRI-compatible robotic system with force feedback for interaction with human motion. IEEE/ASME Trans. Mechatron. 11 (2), 216-224.

Gassert, R., Burdet, E., Chinzei, K., 2008. Opportunities and challenges in MR-compatible robotics. IEEE Eng. Med. Biol. Mag. 27 (3), 15-22.

Gloster, A., Wittchen, H., Einsle, F., Höfler, M., Lang, T., Helbig-Lang, S., Fydrich, T., Fehm, L., Hamm, A., Richter, J., 2009. Mechanism of action in CBT (MAC): methods of a multi-center randomized controlled trial in 369 patients with panic disorder and agoraphobia. Eur. Arch. Psychiatry Clin. Neurosci. 259, 155-166.

Glover, G.H., Li, T.Q., Ress, D., 2000. Image-based method for retrospective correction of physiological motion effects in fMRI: RETROICOR. Magn. Reson. Med. 44 (1), 162-167.

Goebel, R., Sorger, B., Kaiser, J., Birbaumer, N., Weiskopf, N., 2004. BOLD Brain Pong: Self-regulation of Local Brain Activity During Synchronously Scanned, Interacting Subjects (San Diego, CA).

Hajnal, J.V., Myers, R., Oatridge, A., Schwieso, J.E., Young, I.R., Bydder, G.M., 1994. Artifacts due to stimulus correlated motion in functional imaging of the brain. Magn. Reson. Med. 31 (3), 283-291.

Haller, S., Birbaumer, N., Veit, R., 2010. Real-time fMRI feedback training may improve chronic tinnitus. Eur. Radiol. 20 (3), 696-703.

Hamilton, J.P., Glover, G.H., Hsu, J.J., Johnson, R.F., Gotlib, I.H., 2011. Modulation of subgenual anterior cingulate cortex activity with real-time neurofeedback. Human Brain Mapping. 32 (1), 22-31. 
Hawkinson, J.E., Ross, A.J., Parthasarathy, S., Scott, D.J., Laramee, E.A., Posecion, L.J., Rekshan, W.R., Sheau, K.E., Njaka, N.D., Bayley, P.J., DeCharms, R.C., 2012. Quantification of adverse events associated with functional MRI scanning and with real-time fMRIbased training. Int. J. Behav. Med. 19 (3), 372-381.

Hebb, D.O., 1949. The Organization of Behavior: A Neuropsychological Theory. Lawrence Erlbaum.

Hernandez-Garcia, L., Jahanian, H., Greenwald, M.K., Zubieta, J.K., Peltier, S.J., 2011. Real-time functional MRI using pseudo-continuous arterial spin labeling. Magn. Reson. Med. 65 (6), 1570-1577.

Hinds, O., Ghosh, S., Thompson, T.W., Yoo, J.J., Whitfield-Gabrieli, S., Triantafyllou, C., Gabrieli, J.D.E., 2011. Computing moment-to-moment BOLD activation for realtime neurofeedback. NeuroImage 54 (1), 361-368.

Hinds, O., Thompson, T.W., Ghosh, S., Yoo, J.J., Whitfield-Gabrieli, S., Triantafyllou, C., Gabrieli, J.D.E., 2013. Roles of default-mode network and supplementary motor area in human vigilance performance: evidence from real-time fMRI. J. Neurophysiol. 109 (5), 1250-1258.

Hirsch, J., Ruge, M.I., Kim, K.H.S., Correa, D.D., Victor, J.D., Relkin, N.R., Labar, D.R., Krol, G., Bilsky, M.H., Souweidane, M.M., 2000. An integrated functional magnetic resonance imaging procedure for preoperative mapping of cortical areas associated with tactile, motor, language, and visual functions. Neurosurgery 47 (3), 711-722.

Hochberg, L.R., Serruya, M.D., Friehs, G.M., Mukand, J.A., Saleh, M., Caplan, A.H. Branner, A., Chen, D., Penn, R.D., Donoghue, J.P., 2006. Neuronal ensemble control of prosthetic devices by a human with tetraplegia. Nature 442 (7099), 164-171.

Hu, X., Le, T.H., Parrish, T., Erhard, P., 2005. Retrospective estimation and correction of physiological fluctuation in functional MRI. Magn. Reson. Med. 34 (2), 201-212.

Jackson, A., Mavoori, J., Fetz, E.E., 2006. Long-term motor cortex plasticity induced by an electronic neural implant. Nature 444 (7115), 56-60.

Jacobson, N.S., Christensen, A., 1996. Studying the effectiveness of psychotherapy: how well can clinical trials do the job? Am. Psychol. 51 (10), 1031.

Johnston, S.J., Boehm, S.G., Healy, D., Goebel, R., Linden, D.E.J., 2010. Neurofeedback: A promising tool for the self-regulation of emotion networks. Neurolmage. 49 (1), 1066-1072.

Johnston, S., Linden, D.E.J., Healy, D., Goebel, R., Habes, I., Boehm, S.G., 2011. Upregulation of emotion areas through neurofeedback with a focus on positive mood. Cognitive, Affective, \& Behavioral Neuroscience. 11 (1), 44-51.

Johnson, K.A., Hartwell, K., LeMatty, T., Borckardt, J., Morgan, P.S., Govindarajan, K., Brady, K., George, M.S., 2012. Intermittent "real-time" fMRI feedback is superior to continuous presentation for a motor imagery task: a pilot study. J. Neuroimaging $22(1), 58-66$

Kasper, L., Marti, S., Vannesjö, S.J., Hutton, C., Dolan, R., Weiskopf, N., Stephan, K.E., Prüssmann, K.P., 2009. Cardiac Artefact Correction for Human Brainstem fMRI at 7 Tesla. (Proc. Organization of Human Brain Mapping) (San Francisco, CA).

Kim, S.G., Ogawa, S., 2012. Biophysical and physiological origins of blood oxygenation level-dependent fMRI signals. J. Cereb. Blood Flow Metab. 32, 1188-1206.

Kim, S., Rauss, K., Yu, T., Dax, J., Muehlbauer, F., Soekadar, S.R., Birbaumer, N., Sitaram, R., 2011. Training the 6th sense: making subliminal stimuli perceivable using real-time fMRI (Washington, D.C., USA)

Koralek, A.C., Jin, X., Long II, J.D., Costa, R.M., Carmena, J.M., 2012. Corticostriatal plasticity is necessary for learning intentional neuroprosthetic skills. Nature 483 (7389), 331-335.

Kotchoubey, B., Strehl, U., Uhlmann, C., Holzapfel, S., König, M., Fröscher, W., Blankenhorn, V., Birbaumer, N., 2001. Modification of slow cortical potentials in patients with refractory epilepsy: a controlled outcome study. Epilepsia 42 (3), 406-416.

Kotchoubey, B., Lang, S., Mezger, G., Schmalohr, D., Schneck, M., Semmler, A., Bostanov, V., Birbaumer, N., 2005. Information processing in severe disorders of consciousness: vegetative state and minimally conscious state. Clin. Neurophysiol. 116 (10), 2441-2453.

Koush, Y., Elliott, M.A., Mathiak, K., 2011. Single voxel proton spectroscopy for neurofeedback at 7 Tesla. Materials 4 (9), 1548-1563.

Koush, Y., Zvyagintsev, M., Dyck, M., Mathiak, K.A., Mathiak, K., 2012. Signal quality and Bayesian signal processing in neurofeedback based on real-time fMRI. NeuroImage 59 (1), 478-489.

Krüger, G., Glover, G.H., 2001. Physiological noise in oxygenation-sensitive magnetic resonance imaging. Magn. Reson. Med. 46 (4), 631-637.

Kübler, A., Birbaumer, N., 2008. Brain-computer interfaces and communication in paralysis: extinction of goal directed thinking in completely paralysed patients? Clin. Neurophysiol. 119 (11), 2658-2666.

LaConte, S.M., 2011. Decoding fMRI brain states in real-time. NeuroImage 56 (2), 440-454.

LaConte, S.M., Peltier, S.J., Hu, X.P., 2007. Real-time fMRI using brain-state classification. Hum. Brain Mapp. 28 (10), 1033-1044.

Lee, S., Ruiz, S., Caria, A., Veit, R., Birbaumer, N., Sitaram, R., 2011. Detection of cerebral reorganization induced by real-time fMRI feedback training of insula activation a multivariate investigation. Neurorehabil. Neural Repair 25 (3), 259-267.

Lee, J.H., Kim, J., Yoo, S.S., 2012. Real-time fMRI-based neurofeedback reinforces causality of attention networks. Neurosci. Res. 72 (4), 347-354.

Li, X., Hartwell, K.J., Borckardt, J., Prisciandaro, J.J., Saladin, M.E., Morgan, P.S., Johnson, K.A., LeMatty, T., Brady, K.T., George, M.S., 2012. Volitional reduction of anterior cingulate cortex activity produces decreased cue craving in smoking cessation: a preliminary real-time fMRI study. Addict. Biol. http://dx.doi.org/10.1111/j.13691600.2012.00449.

Liberati, G., Birbaumer, N., 2012. Using brain-computer interfaces to overcome the extinction of goal-directed thinking in minimally conscious state patients. Cogn. Process. 13, 239-241.

Liberati, G., da Rocha, J.L.D., van der Heiden, L., Raffone, A., Birbaumer, N., Olivetti Belardinelli, M., Sitaram, R., 2012. Toward a brain-computer interface for
Alzheimer's disease patients by combining classical conditioning and brain state classification. J. Alzheimers Dis. 31, 211-220.

Linden, D.E.J., 2012a. The challenges and promise of neuroimaging in psychiatry. Neuron 73 (1), 8-22.

Linden, D.E., 2012b. The Biology of Psychological Disorders. Palgrave Macmillan.

Linden, D.E.J., Habes, I., Johnston, S.J., Linden, S., Tatineni, R., Subramanian, L., Sorger, B. Healy, D., Goebel, R., 2012. Real-time self-regulation of emotion networks in patients with depression. PLoS One 7 (6), e38115.

Logothetis, N.K., 2008. What we can do and what we cannot do with fMRI. Nature 453 (7197), 869-878.

Logothetis, N.K., Pauls, J., Augath, M., Trinath, T., Oeltermann, A., 2001. Neurophysiological investigation of the basis of the fMRI signal. Nature 412 (6843), 150-157.

Lynch, J.J., Paskewitz, D.A., Orne, M.T., 1974. Some factors in the feedback control of human alpha rhythm. Psychosom. Med. 36 (5), 399-410.

Martin, S., Morris, R., 2002. New life in an old idea: the synaptic plasticity and memory hypothesis revisited. Hippocampus 12 (5), 609-636.

McCaig, R.G., Dixon, M., Keramatian, K., Liu, I., Christoff, K., 2011. Improved modulation of rostrolateral prefrontal cortex using real-time fMRI training and meta-cognitive awareness. NeuroImage. 55 (3), 1298-1305.

Miller, N.E., 1975. Learning of visceral and glandular responses. Development of psycho-motor competence: selected readings, 163, p. 114

Miller, N.E., Dworkin, B.K., 1974. Visceral learning: recent difficulties with curarized rats and significant problems for human research. In: Obrist, Black, Brener, Jasper, DiCara, Leo (Eds.), AldineTransaction, New Brunswick, NJ.

Montague, P.R., Berns, G.S., Cohen, J.D., McClure, S.M., Pagnoni, G., Dhamala, M., Wiest, M.C., Karpov, I., King, R.D., Apple, N., 2002. Hyperscanning: simultaneous fMRI during linked social interactions. NeuroImage 16 (4), 1159-1164.

Monti, M.M., Vanhaudenhuyse, A., Coleman, M.R., Boly, M., Pickard, J.D., Tshibanda, L. Owen, A.M., Laureys, S., 2010. Willful modulation of brain activity in disorders of consciousness. N. Engl. J. Med. 362 (7), 579-589.

Moore, C.I., Cao, R., 2008. The hemo-neural hypothesis: on the role of blood flow in information processing. J. Neurophysiol. 99 (5), 2035-2047.

Moritz, C.T., Perlmutter, S.I., Fetz, E.E., 2008. Direct control of paralysed muscles by cortical neurons. Nature 456 (7222), 639-642.

Naito, M., Michioka, Y., Ozawa, K., Kiguchi, M., Kanazawa, T., 2007. A communication means for totally locked-in ALS patients based on changes in cerebral blood volume measured with near-infrared light. IEICE transactions on information and systems 90 (7), 1028-1037.

Ochsner, K.N., Silvers, J.A., Buhle, J.T., 2012. Functional imaging studies of emotion regulation: a synthetic review and evolving model of the cognitive control of emotion. Ann. N. Y. Acad. Sci. 1251 (1), E1-E24.

Ogawa, S., Lee, T., Kay, A., Tank, D., 1990a. Brain magnetic resonance imaging with contrast dependent on blood oxygenation. Proc. Natl. Acad. Sci. 87 (24), 9868-9872.

Ogawa, S., Lee, T.M., Nayak, A.S., Glynn, P., 1990b. Oxygenation-sensitive contrast in magnetic resonance image of rodent brain at high magnetic fields. Magn. Reson. Med. 14 (1), 68-78.

Olds, J., 1965. Operant Conditioning of Single Unit Responses, pp. 1-9 (Tokyo, Japan).

Öst, L.G., Westling, B.E., 1995. Applied relaxation vs cognitive behavior therapy in the treatment of panic disorder. Behav. Res. Ther. 33 (2), 145-158.

Phillips, M., Ladouceur, C., Drevets, W., 2008. A neural model of voluntary and automatic emotion regulation: implications for understanding the pathophysiology and neurodevelopment of bipolar disorder. Mol. Psychiatry 13 (9), 833-857.

Plotkin, A., Sela, L., Weissbrod, A., Kahana, R., Haviv, L., Yeshurun, Y., Soroker, N., Sobel, N., 2010. Sniffing enables communication and environmental control for the severely disabled. Proc. Natl. Acad. Sci. 107 (32), 14413-14418.

Posse, S., Fitzgerald, D., Gao, K., Habel, U., Rosenberg, D., Moore, G.J., Schneider, F., 2003. Real-time fMRI of temporolimbic regions detects amygdala activation during single-trial self-induced sadness. Neurolmage 18 (3), 760-768.

Posse, S., Ackley, E., Mutihac, R., Rick, J., Shane, M., Murray-Krezan, C., Zaitsev, M., Speck O., 2012. Enhancement of temporal resolution and BOLD sensitivity in real-time fMRI using multi-slab echo-volumar imaging. Neurolmage 61 (1), 115-130.

Ramos Murguialday, A., Halder, S., Birbaumer, N., 2009. Proprioceptive Feedback in BCI

Rockstroh, B., Birbaumer, N., Elbert, T., Lutzenberger, W., 1984. Operant control of EEG and event-related and slow brain potentials. Appl. Psychophysiol. Biofeedback 9 (2), 139-160.

Rockstroh, B., Elbert, T., Birbaumer, N., Wolf, P., Düchting-Röth, A., Reker, M., Daum, I., Lutzenberger, W., Dichgans, J., 1993. Cortical self-regulation in patients with epilepsies. Epilepsy Res. 14 (1), 63-72.

Roebroeck, A., Formisano, E., Goebel, R., 2005. Mapping directed influence over the brain using Granger causality and fMRI. NeuroImage 25 (1), 230-242.

Rota, G., Sitaram, R., Veit, R., Erb, M., Weiskopf, N., Dogil, G., Birbaumer, N., 2009. Selfregulation of regional cortical activity using real-time fMRI: the right inferior frontal gyrus and linguistic processing. Hum. Brain Mapp. 30 (5), 1605-1614.

Rota, G., Handjaras, G., Sitaram, R., Birbaumer, N., Dogil, G., 2011. Reorganization of functional and effective connectivity during real-time fMRI-BCI modulation of prosody processing. Brain Lang. 117 (3), 123-132.

Ruiz, S., Lee, S., Soekadar, S.R., Caria, A., Veit, R., Kircher, T., Birbaumer, N., Sitaram, R. 2013. Acquired self-control of insula cortex modulates emotion recognition and brain network connectivity in schizophrenia. Hum. Brain Mapp. 34 (1), 200-212.

Schaefer, D.J., Bourland, J.D., Nyenhuis, J.A., 2000. Review of patient safety in timevarying gradient fields. J. Magn. Reson. Imaging 12 (1), 20-29.

Schafer, R.J., Moore, T., 2011. Selective attention from voluntary control of neurons in prefrontal cortex. Science 332 (6037), 1568-1571.

Scharnowski, F., Hutton, C., Josephs, O., Weiskopf, N., Rees, G., 2012. Improving visual perception through neurofeedback. J. Neurosci. 32 (49), 17830-17841.

Schenck, J.F., 2000. Safety of strong, static magnetic fields. J. Magn. Reson. Imaging 12 (1), 2-19. 
Schwartz, M.S., Andrasik, F., 2003. Biofeedback: A Practitioner's Guide. Guilford Press. Schwartz, G.E., Beatty, J., 1977. Biofeedback, Theory and Research. Academic Press.

Shellock, F.G., Crues, J.V., 2004. MR procedures: biologic effects, safety, and patient care. Radiology 232 (3), 635-652.

Shibata, K., Watanabe, T., Sasaki, Y., Kawato, M., 2011. Perceptual learning incepted by decoded fMRI neurofeedback without stimulus presentation. Science 334 (6061), 1413-1415.

Sitaram, R., Caria, A., Veit, R., Gaber, T., Kuebler, A., Birbaumer, N., 2005. Real-time fMR Based Brain-Computer Interface Enhanced by Interactive Virtual Worlds (Lisbon, Portugal).

Sitaram, R., Zhang, H., Guan, C., Thulasidas, M., Hoshi, Y., Ishikawa, A., Shimizu, K. Birbaumer, N., 2007. Temporal classification of multichannel near-infrared spectroscopy signals of motor imagery for developing a brain-computer interface. Neurolmage 34 (4), 1416-1427.

Sitaram, R., Lee, S., Ruiz, S., Birbaumer, N., 2010. Real-time regulation and detection of brain states from fMRI signals. Neurofeedback and Neuromodulation Techniques and Applications, p. 227.

Sitaram, R., Lee, S., Ruiz, S., Rana, M., Veit, R., Birbaumer, N., 2011. Real-time support vector classification and feedback of multiple emotional brain states. Neurolmage 56 (2), 753-765.

Sitaram, R., Veit, R., Stevens, B., Caria, A., Gerloff, C., Birbaumer, N., Hummel, F., 2012. Acquired control of ventral premotor cortex activity by feedback training an exploratory real-time fMRI and TMS study. Neurorehabil. Neural Repair 26 (3), 256-265.

Sorger, B., Reithler, J., Dahmen, B., Goebel, R., 2012. A real-time fMRI-based spelling device immediately enabling robust motor-independent communication. Curr. Biol. 22 (14), 1333-1338.

Stroebel, C.F., Glueck, B.C., 1973. Biofeedback Treatment in Medicine and Psychiatry: An Ultimate Placebo? Grune \& Stratton, Inc.

Subramanian, L., Hindle, J.V., Johnston, S., Roberts, M.V., Husain, M., Goebel, R., Linden, D., 2011. Real-time functional magnetic resonance imaging neurofeedback for treatment of Parkinson's disease. J. Neurosci. 31 (45), 16309-16317.

Sulzer, J., Sitaram, R., Blefari, M.L., Birbaumer, N., Kollias, S., Stephan, K.E., Luft, A Gassert, R., in press. Neurofeedback-mediated self-regulation of the dopaminergic midbrain. Neurolmage.

Vaadia, E., Birbaumer, N., 2009. Grand challenges of brain computer interfaces in the years to come. Frontiers in neuroscience 3 (2), 151.

Veit, R., Singh, V., Sitaram, R., Caria, A., Rauss, K., Birbaumer, N., 2012. Using real-time fMRI to learn voluntary regulation of the anterior insula in the presence of threat-related stimuli. Soc. Cogn. Affect. Neurosci. 7 (6), 623-634.
Wald, L.L., 2012. The future of acquisition speed, coverage, sensitivity, and resolution. NeuroImage 62 (2), 1221-1229.

Weiskopf, N., 2012. Real-time fMRI and its application to neurofeedback. Neurolmage $62(2), 682$.

Weiskopf, N., Veit, R., Erb, M., Mathiak, K., Grodd, W., Goebel, R., Birbaumer, N., 2003. Physiological self-regulation of regional brain activity using real-time functional magnetic resonance imaging (fMRI): methodology and exemplary data. Neurolmage 19 (3), 577-586.

Weiskopf, N., Mathiak, K., Bock, S.W., Scharnowski, F., Veit, R., Grodd, W., Goebel, R., Birbaumer, N., 2004a. Principles of a brain-computer interface (BCI) based on real-time functional magnetic resonance imaging (fMRI). IEEE Trans. Biomed. Eng. 51 (6), 966-970.

Weiskopf, N., Scharnowski, F., Veit, R., Goebel, R., Birbaumer, N., Mathiak, K., 2004b. Self-regulation of local brain activity using real-time functional magnetic resonance imaging (fMRI). J. Physiol. Paris 98 (4-6), 357-373.

Weiskopf, N., Sitaram, R., Josephs, O., Veit, R., Scharnowski, F., Goebel, R., Birbaumer, N., Deichmann, R., Mathiak, K., 2007. Real-time functional magnetic resonance imaging: methods and applications. Magn. Reson. Imaging 25 (6), 989-1003.

Wilhelm, B., Jordan, M., Birbaumer, N., 2006. Communication in locked-in syndrome: effects of imagery on salivary pH. Neurology 67 (3), 534-535.

Wyrwicka, W., Sterman, M.B., 1968. Instrumental conditioning of sensorimotor cortex EEG spindles in the waking cat. Physiol. Behav. 3 (5), 703-707.

Yoo, S.S., Jolesz, F.A., 2002. Functional MRI for neurofeedback: feasibility study on a hand motor task. Neuroreport 13 (11), 1377.

Yoo, S.S., Fairneny, T., Chen, N.K., Choo, S.E., Panych, L.P., Park, H.W., Lee, S.Y., Jolesz, F.A., 2004. Brain-computer interface using fMRI: spatial navigation by thoughts. Neuroreport 15 (10), 1591

Yoo, S.S., Lee, J.H., O'Leary, H., Panych, L.P., Jolesz, F.A., 2008. Neurofeedback fMRImediated learning and consolidation of regional brain activation during motor imagery. International Journal of Imaging Systems and Technology. 18 (1), 69-78.

Yoo, J.J., Hinds, O., Ofen, N., Thompson, T.W., Whitfield-Gabrieli, S., Triantafyllou, C., Gabrieli, J.D.E., 2012. When the brain is prepared to learn: enhancing human learning using real-time fMRI. NeuroImage 59 (1), 846-852.

Zhang, X., Ross, T.J., Jo Salmeron, B., Yang, S., Yang, Y., Stein, E.A., 2011. Single subject task-related BOLD signal artifact in a real-time fMRI feedback paradigm. Hum. Brain Mapp. 32 (4), 592-600.

Zotev, V., Krueger, F., Phillips, R., Alvarez, R.P., Simmons, W.K., Bellgowan, P., Drevets, W.C., Bodurka, J., 2011. Self-regulation of amygdala activation using real-time fMRI neurofeedback. PloS One. 6 (9), e24522. 